\title{
Capacity of a POST Channel with and without Feedback
}

\author{
Haim H. Permuter, Himanshu Asnani and Tsachy Weissman
}

\begin{abstract}
We consider finite state channels where the state of the channel is its previous output. We refer to these as POST (Previous Output is the STate) channels. We first focus on $\operatorname{POST}(\alpha)$ channels. These channels have binary inputs and outputs, where the state determines if the channel behaves as a $Z$ or an $S$ channel, both with parameter $\alpha$. We show that the non feedback capacity of the $\operatorname{POST}(\alpha)$ channel equals its feedback capacity, despite the memory of the channel. The proof of this surprising result is based on showing that the induced output distribution, when maximizing the directed information in the presence of feedback, can also be achieved by an input distribution that does not utilize of the feedback. We show that this is a sufficient condition for the feedback capacity to equal the non feedback capacity for any finite state channel. We show that the result carries over from the POST $(\alpha)$ channel to a binary POST channel where the previous output determines whether the current channel will be binary with parameters $(a, b)$ or $(b, a)$. Finally, we show that, in general, feedback may increase the capacity of a POST channel.
\end{abstract}

\section{Index Terms}

Causal conditioning, Convex optimization, Channels with memory, Directed information, Feedback capacity, Finite state channel, KKT conditions, POST channel.

\section{INTRODUCTION}

The capacity of a memoryless channel is very well understood. There are many simple memoryless channels for which we know the capacity analytically. These include the binary symmetric channel, the erasure channel, the additive Gaussian channel and the $Z$ Channel. Furthermore, using convex optimization tools, such as the BlahutArimoto algorithm [1], [2], we can efficiently compute the capacity of any memoryless channel with a finite alphabet. However, in the case of channels with memory, the exact capacities are known for only a few channels, such as additive Gaussian channels (water filling solution) [3], [4] and discrete additive channels with memory [5]. In cases where feedback is allowed, there are only a few more cases where the exact capacity is known, such as the moduloadditive noise channel, the additive noise channel where the noise is a first-order autoregressive moving-average Gaussian process [6], the trapdoor channel [7], and the Ising Channel [8]. If the state is known at the decoder, then knowledge of the state at the encoder can be considered as partial feedback, as considered and solved in [9] and in [10].

H. Permuter is with the department of Electrical and Computer Engineering, Ben-Gurion University of the Negev, Beer-Sheva, Israel (haimp@bgu.ac.il). H. Asnani and T. Weissman are with the Department of Electrical Engineering, Stanford University, CA, USA (asnani@stanford.edu, tsachy@stanford.edu). This paper was presented in part at the 2013 IEEE International Symposium on Information Theory. 

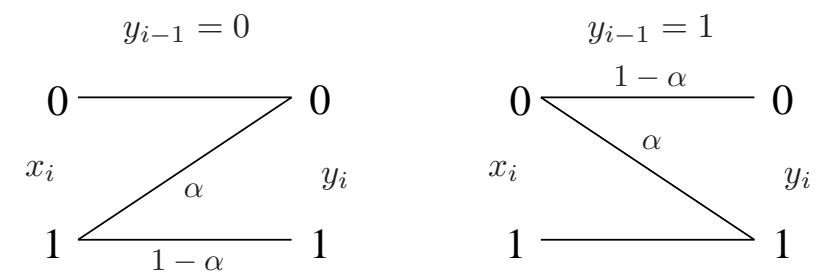

Fig. 1. $\operatorname{POST}(\alpha)$ : If $y_{i-1}=0$ then the channel behaves as a $Z$ channel with parameter $\alpha$ and if $y_{i-1}=1$ then it behaves as an $S$ channel with parameter $\alpha$.

In this paper we introduce and consider a new family of channels that we refer to as "POST channels". These are simple Finite State Channels (FSCs) where the state of the channel is the previous output. In particular, we focus on a family of POST channels that have binary inputs $\left\{X_{i}\right\}_{i \geq 1}$ and binary outputs $\left\{Y_{i}\right\}_{i \geq 1}$ related as follows:

$$
\text { if } X_{i}=Y_{i-1} \text {, then } Y_{i}=X_{i} \text {, else } Y_{i}=X_{i} \oplus Z_{i} \text {, where } Z_{i} \sim \operatorname{Bernnouli}(\alpha) \text {. }
$$

We call these channels $\operatorname{POST}(\alpha)$ and their behavior is depicted in Fig. 11 When $y_{i-1}=0$, the current channel behaves as a $Z$ channel with parameter $\alpha$ and when $y_{i-1}=1$, it behaves as an $S$ channel with parameter $\alpha$. We refer to $\operatorname{POST}\left(\frac{1}{2}\right)$ as the simple POST channel.

The simple POST channel is similar to the Ising channel introduced by Berger and Bonomi [11], but rather than the previous input being the state of the channel, here the state of the channel is the previous output. This channel arose in the investigation of controlled feedback in the setting of "to feed or not to feed back" [12]. The POST channel can also be useful in modeling memory affected by past channel outputs, as is the case in flash memory and other storage devices.

In order to gain intuition for investigating the influence of feedback on the simple POST channel, let us first consider a channel with binary i.i.d. states $S_{i}$, distributed Bernoulli $\left(\frac{1}{2}\right)$, where the channel behaves similarly to the simple POST channel. When $S_{i-1}=0$, then the current channel behaves as a $Z$ channel and when $S_{i-1}=1$, it behaves as an $S$ channel, as shown in Fig. 2. Similar to the POST channel, we assume that the state of the channel is known to the decoder; hence the output of the channel is $\left(Y_{i}, S_{i}\right)$ (or, equivalently from a capacity standpoint, $\left.\left(Y_{i}, S_{i-1}\right)\right)$.

The non feedback capacity of this channel is simply $C=\max _{P(x)} I(X ; Y, S)$ and, because of symmetry, the input that achieves the maximum is Bernoulli $\left(\frac{1}{2}\right)$, resulting in a capacity of $H_{b}\left(\frac{1}{4}\right)-\frac{1}{2}=0.3111$, where $H_{b}(p)$ is the binary entropy function. However, if there is perfect feedback of $\left(Y_{i}, S_{i}\right)$ to the encoder, then the state of the channel is known to the encoder and the capacity is simply the capacity of the $Z$ (or $S$ ) channel, which is $H_{b}\left(\frac{1}{5}\right)-\frac{2}{5}=-\log _{2} 0.8=0.3219$. Evidently, feedback increases the capacity of this channel.

The similarity between the channels may seem to hint that feedback increases the capacity of the POST $(\alpha)$ channel as well. Indeed, our initial interest in this channel was due to this belief, in our quest for a channel with memory that would be amenable to analysis under the "to feed or not to feed" framework of constrained feedback 


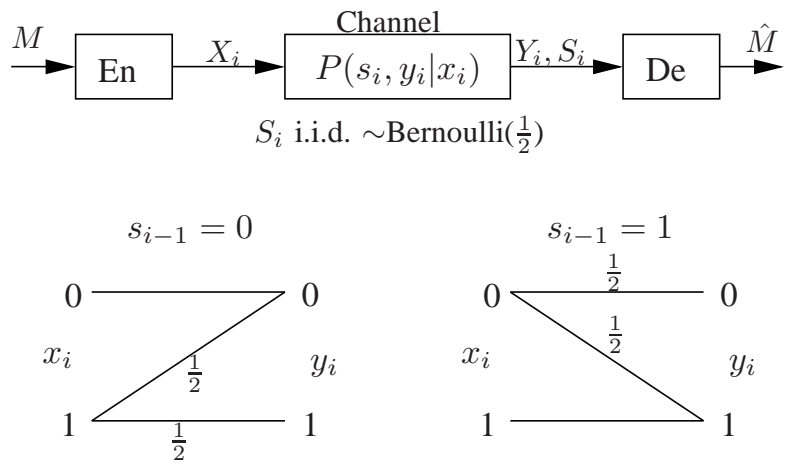

Fig. 2. A channel similar to the simple POST channel, except that the channel state, $\left\{S_{i}\right\}_{i \geq 1}$ is i.i.d. Bernoulli( $\left.\frac{1}{2}\right)$ independent of the input.

in [12], while exhibiting non-trivial dependence of its capacity on the extent to which the feedback is constrained. However, numerical results based on the computational algorithm devised in [12] suggested that feedback does not increase the capacity of the simple POST channel. This paper stemmed from our attempts to make sense of these observations.

In order to prove that feedback does not increase the capacity of some families of POST channels, we look at two convex optimization problems: maximizing the directed information over regular input distributions (non feedback case), i.e., $P\left(x^{n}\right)$ and, secondly, over causal conditioning that is influenced by the feedback i.e., $P\left(x^{n} \| y^{n-1}\right)$. We show that a necessary and sufficient condition for the solutions of the two optimization problems to achieve the same value is that the induced output distributions $P\left(y^{n}\right)$ by the respective optimal values $P^{*}\left(x^{n}\right)$ and $P^{*}\left(x^{n} \| y^{n-1}\right)$ are the same. This necessary and sufficient condition that we establish, in the generality of any finite state channel, follows from the KKT conditions [13, Ch. 5] for convex optimization problems.

The remainder of the paper is organized as follows. In Section [1] we briefly present the definitions of directed information and causal conditioning pmfs that we use throughout the paper. In Section III we show that the optimization problem of maximizing the directed information over causal conditioning pmfs is convex. Additionally, using the KKT conditions, we show that if the output distribution induced by the conditional pmfs that achieve the maximum in the presence of feedback can also be induced by an input distribution that does not use feedback, then feedback does not increase the capacity. In Section IV we compute the feedback capacity of the POST $(\alpha)$ channel. Then we apply the result of Section 【III to show that it equals the non feedback capacity by establishing the existence of an input distribution without feedback that induces the same output distribution as the capacity achieving one for the feedback case. In Section $\nabla$ we consider a binary $\operatorname{POST}(a, b)$ channel with two states; in each state there is a binary channel and the channels have opposite parameters. The binary $\operatorname{POST}(a, b)$ channel generalizes the $\operatorname{POST}(\alpha)$ channel and we show that feedback does not increase capacity for this considerably larger class of channels. In Section VI, we show that unlike the the POST $(a, b)$, feedback may increase the capacity of POST channels in general. In Section VII] we conclude and suggest some directions for further research on the family of POST channels. 


\section{DiRECTED INFORMATION, CAUSAL CONDITIONING AND NOTATIONS}

Throughout this paper, we denote random variables by capital letters such as $X$. The probability $\operatorname{Pr}\{X=x\}$ is denoted by $p(x)$. We denote the whole vector of probabilities by capital $P$, i.e., $P(x)$ is the probability vector of the random variable $X$.

We use the causal conditioning notation $(\cdot \| \cdot)$ developed by Kramer [14]. We denote by $p\left(x^{n} \| y^{n-d}\right)$ the probability mass function of $X^{n}=\left(X_{1}, \ldots, X_{n}\right)$, causally conditioned on $Y^{n-d}$ for some integer $d \geq 0$, which is defined as

$$
p\left(x^{n}|| y^{n-d}\right):=\prod_{i=1}^{n} p\left(x_{i} \mid x^{i-1}, y^{i-d}\right) .
$$

By convention, if $i<d$, then $y^{i-d}$ is set to null, i.e., if $i<d$ then $p\left(x_{i} \mid x^{i-1}, y^{i-d}\right)$ is just $p\left(x_{i} \mid x^{i-1}\right)$. In particular, we use extensively the cases $d=0,1$ :

$$
\begin{aligned}
p\left(x^{n} \| y^{n}\right) & :=\prod_{i=1}^{n} p\left(x_{i} \mid x^{i-1}, y^{i}\right), \\
p\left(x^{n} \| y^{n-1}\right) & :=\prod_{i=1}^{n} p\left(x_{i} \mid x^{i-1}, y^{i-1}\right) .
\end{aligned}
$$

The directed information was defined by Massey [15], inspired by Marko's work [16] on bidirectional communication, as

$$
I\left(X^{n} \rightarrow Y^{n}\right):=\sum_{i=1}^{n} I\left(X^{i} ; Y_{i} \mid Y^{i-1}\right) .
$$

The directed information can also be rewritten as

$$
I\left(X^{n} \rightarrow Y^{n}\right)=\sum_{x^{n}, y^{n}} p\left(x^{n} \| y^{n-1}\right) p\left(y^{n} \| x^{n}\right) \log \frac{p\left(y^{n} \| x^{n}\right)}{\sum_{x^{n}} p\left(x^{n} \| y^{n-1}\right) p\left(y^{n} \| x^{n}\right)}
$$

This is due to the definition of causal conditioning and the chain rule

$$
p\left(x^{n}, y^{n}\right)=p\left(x^{n} \| y^{n-1}\right) p\left(y^{n} \| x^{n}\right)
$$

We will make use the fact that directed information $I\left(X^{n} \rightarrow Y^{n}\right)$ is concave in $P\left(x^{n} \| y^{n-1}\right)$ for a fixed $P\left(y^{n} \| x^{n}\right)$, which is proved in Lemma 16 in Appendix A

Directed information characterizes the capacity of point-to-point channels with feedback [10], [17]-[19]. For channels where the state is a function of the output, of which the POST channel is a special case, it was shown [7], [10] that the feedback capacity is given by

$$
C_{f b}=\lim _{n \rightarrow \infty} \frac{1}{n} \max _{P\left(x^{n} \| y^{n-1}\right)} I\left(X^{n} \rightarrow Y^{n}\right) .
$$

On the other hand, without feedback the capacity is given by

$$
C=\lim _{n \rightarrow \infty} \frac{1}{n} \max _{P\left(x^{n}\right)} I\left(X^{n} \rightarrow Y^{n}\right),
$$

since the channel is indecomposable [20]. In the case where there is no feedback, namely, the Markov form $X_{i}-X^{i-1}-Y^{i-1}$ holds, $I\left(X^{n} \rightarrow Y^{n}\right)=I\left(X^{n} ; Y^{n}\right)$, as shown in [15]. 


\section{MAXimizATION OF THE DiRECTED INFORMATION AS A CONVEX OPTIMIZATION PROBLEM}

In order to show that feedback does not increase the capacity of POST channels, we consider the two optimization problems:

$$
\max _{P\left(x^{n} \| y^{n-1}\right)} I\left(X^{n} \rightarrow Y^{n}\right)
$$

and

$$
\max _{P\left(x^{n}\right)} I\left(X^{n} \rightarrow Y^{n}\right)
$$

In this section, we show that both problems are convex optimization problems, and use the KKT condition to state a necessary and sufficient condition for the two optimization problems to obtain the same value.

A convex optimization problem, as defined in [13, Ch. 4], is a problem of the form

$$
\begin{array}{ll}
\text { minimize } & f_{0}(x) \\
\text { subject to } & f_{i}(x) \leq b_{i} \quad i=1, \cdots, k \\
& g_{j}(x)=0 \quad j=1, \cdots, l
\end{array}
$$

where $f_{0}(x)$ and $\left\{f_{i}(x)\right\}_{i=1}^{k}$ are convex functions, and $\left\{g_{j}(x)\right\}_{j=1}^{l}$ are affine.

In order to convert the optimization problem in (10) into a convex optimization problem, as presented in (12), we need to show that the set of conditional pmfs $P\left(x^{n} \| y^{n-1}\right)$ can be expressed using inequalities that contains only convex functions and equalities that contains affine functions.

Lemma 1 (Causal conditioning is a polyhedron): The set of all causal conditioning distributions of the form $P\left(x^{n}|| y^{n-1}\right)$ is a polyhedron in $\mathbb{R}^{|\mathcal{X}|^{n}|\mathcal{Y}|^{n-1}}$ and is given by a set of linear equalities and inequalities of the form:

$$
\begin{array}{ll}
p\left(x^{n} \| y^{n-1}\right) \geq 0, & \forall x^{n}, y^{n-1}, \\
\sum_{x_{i+1}^{n}} p\left(x^{n} \| y^{n-1}\right)=\gamma_{x^{i}, y^{i-1}}, & \forall x^{i}, y^{n-1}, i \geq 1, \\
\sum_{x_{1}^{n}} p\left(x^{n} \| y^{n-1}\right)=1, & \forall y^{n-1} .
\end{array}
$$

Note that the two equalities in (13) may be unified into one if we add $i=0$ to the equality cases and we restrict the corresponding $\gamma$ to be unity. Furthermore, for $n=1$ we obtain the regular vector probability, i.e., $p(x) \geq 0, \forall x$ and $\sum_{x} P(x)=1$.

Proof: It is straightforward to see that every causal conditioning $P\left(x^{n} \| y^{n}\right)$ satisfies these equalities and inequalities. Now, we need to show that if an element in $\mathbb{R}^{|\mathcal{X}|^{n}|\mathcal{Y}|^{n-1}}$, call it $P\left(x^{n}|| y^{n-1}\right)$, satisfies 13 , then $P\left(x^{n}|| y^{n-1}\right)$ is a causal conditioning pmf, namely, there exists a sequence of regular conditioning $\left\{P\left(x_{i} \mid x^{i-1}, y^{i-1}\right)\right\}_{i=1}^{n}$ such that $p\left(x^{n} \| y^{n-1}\right)=\prod_{i=1}^{n} p\left(x_{i} \mid x^{i-1}, y^{i-1}\right)$.

Let us define for all $x^{i}, y^{i-1}$

$$
\begin{aligned}
p\left(x_{i} \mid x^{i-1}, y^{i-1}\right) & \triangleq \frac{\sum_{x^{i+1}}^{n} p\left(x^{n} \| y^{n}\right)}{\sum_{x^{i}}^{n} p\left(x^{n} \| y^{n}\right)} \\
& =\frac{\gamma_{x^{i}, y^{i-1}}}{\gamma_{x^{i-1}, y^{i-2}}} .
\end{aligned}
$$


Now, note that the vector probability $P\left(x_{i} \mid x^{i-1}, y^{i-1}\right)$ defined in (14) satisfies $p\left(x_{i} \mid x^{i-1}, y^{i-1}\right) \geq 0$, and $\sum_{x_{i}} p\left(x_{i} \mid x^{i-1}, y^{i-1}\right)=1$. Furthermore, observe that

$$
\prod_{i=1}^{n} p\left(x_{i} \mid x^{i-1}, y^{i-1}\right)=\prod_{i=1}^{n} \frac{\gamma_{x^{i}, y^{i-1}}}{\gamma_{x^{i-1}, y^{i-2}}}=\frac{\gamma_{x^{n}, y^{n-1}}}{1}=\gamma_{x^{n}, y^{n-1}}=p\left(x^{n} \| y^{n-1}\right) .
$$

Note that the optimization problem given in 10 is a convex optimization one since the set of causal conditioning pmfs is a polyhedron (Lemma 1) and the directed information is concave in $P\left(x^{n} \| y^{n-1}\right)$ for a fixed $P\left(y^{n} \| x^{n}\right)$ [21, Lemma 2]. Therefore, the KKT conditions [13, Ch 5.5.3] are necessary and sufficient. The next theorem states these conditions explicitly for our setting.

Theorem 2 (Necessary and sufficient conditions for maximizing the the directed information): A set of necessary and sufficient conditions for an input probability $P\left(x^{n} \| y^{n-1}\right)$ to achieve the maximum in (9) is that for some numbers $\beta_{y^{n-1}}$

$$
\begin{aligned}
& \sum_{y_{n}} p\left(y^{n} \| x^{n}\right) \log \frac{p\left(y^{n} \| x^{n}\right)}{e p\left(y^{n}\right)}=\beta_{y^{n-1}}, \quad \forall x^{n}, y^{n-1}, \text { if } p\left(x^{n} \| y^{n-1}\right)>0 \\
& \sum_{y_{n}} p\left(y^{n} \| x^{n}\right) \log \frac{p\left(y^{n} \| x^{n}\right)}{e p\left(y^{n}\right)} \leq \beta_{y^{n-1}}, \quad \forall x^{n}, y^{n-1}, \text { if } p\left(x^{n} \| y^{n-1}\right)=0
\end{aligned}
$$

where $p\left(y^{n}\right)=\sum_{x^{n}} p\left(y^{n} \| x^{n}\right) p\left(x^{n} \| y^{n-1}\right)$. Furthermore, the maximum is given by

$$
\max _{P\left(x^{n} \| y^{n-1}\right)} I\left(X^{n} \rightarrow Y^{n}\right)=\sum_{y^{n-1}} \beta_{y^{n-1}}+1 .
$$

For $n=1$ we obtain a known result proved by Gallager [20, Theorem 4.5.1] that states that a sufficient and necessary condition for $P^{*}(x)$ to achieve $\max _{P(x)} I(X ; Y)$ is that

$$
\sum_{y} p(y \mid x) \log \frac{p(y \mid x)}{p(y)}=C, \quad \forall x \text { if } p^{*}(x)>0,
$$

and

$$
\sum_{y} p(y \mid x) \log \frac{p(y \mid x)}{p(y)} \leq C, \quad \forall x \text { if } p(x)=0,
$$

for some $C$. Furthermore, $C=\max _{P(x)} I(X ; Y)$.

Proof: Using the fact that a causal conditioning pmf is a polyhedron (Lemma 1), we can write the maximization of the directed information as a standard convex optimization problem:

$$
\begin{array}{ll}
\operatorname{minimize} & -\sum_{x^{n}, y^{n}} p\left(x^{n} \| y^{n-1}\right) p\left(y^{n} \| x^{n}\right) \log \frac{p\left(y^{n} \| x^{n}\right)}{\sum_{x^{n}} p\left(x^{n} \| y^{n-1}\right) p\left(y^{n} \| x^{n}\right)} \\
\text { s.t. } & -p\left(x^{n} \| y^{n-1}\right) \leq 0, \forall x^{n}, y^{n-1}, \\
& \sum_{x_{i+1}^{n}} p\left(x^{n} \| y^{n-1}\right)-\gamma_{x^{i}}, y^{i-1}=0, \forall x^{i}, y^{n-1}, i \geq 1 \\
& \sum_{x^{n}} p\left(x^{n} \| y^{n-1}\right)=1, \forall y^{n-1} .
\end{array}
$$

The Lagrangian is defined as

$$
L=-\sum_{x^{n}, y^{n}} p\left(x^{n} \| y^{n-1}\right) p\left(y^{n} \| x^{n}\right) \log \frac{p\left(y^{n} \| x^{n}\right)}{\sum_{x^{n}} p\left(x^{n} \| y^{n-1}\right) p\left(y^{n} \| x^{n}\right)}-\sum_{x^{n}, y^{n-1}} \lambda_{x^{n}, y^{n-1}} p\left(x^{n} \| y^{n-1}\right)
$$




$$
+\sum_{i=0}^{n} \sum_{x^{i}, y^{n-1}} \nu_{x^{i}, y^{n-1}}\left(\sum_{x_{i+1}^{n}} p\left(x^{n} \| y^{n-1}\right)-\gamma_{x^{i}, y^{i-1}}\right)+\sum_{y^{n-1}} \nu_{y^{n-1}}\left(\sum_{x^{n}} p\left(x^{n} \| y^{n-1}\right)-1\right),
$$

where $\lambda_{x^{n}, y^{n-1}} \geq 0$. The KKT conditions for this problem are

$$
\begin{aligned}
\frac{\partial L}{\partial L p\left(x^{n} \| y^{n-1}\right)} & =0, \forall x^{n}, y^{n-1}, \\
\frac{\partial L}{\partial L \gamma_{x^{i}, y^{i-1}}} & =0, \forall x^{n}, y^{n-1}, \\
\lambda_{x^{n}, y^{n-1}} & \geq 0, \forall x^{n}, y^{n-1}, \\
\lambda_{x^{n}, y^{n-1}} p\left(x^{n} \| y^{n-1}\right) & =0, \forall x^{n}, y^{n-1}
\end{aligned}
$$

and that $P\left(x^{n} \| y^{n-1}\right)$ is a valid causal conditioning pmf, namely, satisfies the constraint of [19]. Now, we need to show that the KKT conditions described above are equivalent to 15 b. Let us compute the derivatives in order to write the conditions in (21) more explicitly:

$$
\begin{aligned}
& \frac{\partial L}{\partial p\left(x^{n} \| y^{n-1}\right)}=-\sum_{y_{n}} p\left(y^{n} \| x^{n}\right) \log P\left(y^{n} \| x^{n}\right)-\sum_{y_{n}} p\left(y^{n} \| x^{n}\right) \log \frac{1}{\sum_{x^{n}} p\left(x^{n} \| y^{n-1}\right) P\left(y^{n} \| x^{n}\right)} \\
&+\sum_{x^{\prime n}, y_{n}} p\left(x^{\prime n} \| y^{n-1}\right) P\left(y^{n} \| x^{\prime n}\right) \frac{1}{\sum_{\tilde{x}^{n}} p\left(\tilde{x}^{n} \| y^{n-1}\right) p\left(y^{n} \| \tilde{x}^{n}\right)} p\left(y^{n} \| x^{n}\right) \\
&-\lambda_{x^{n}, y^{n-1}}+\sum_{i=0}^{n} \nu_{x^{i}, y^{n-1}}+\nu_{y^{n-1}} \\
&=-\sum_{y_{n}} p\left(y^{n} \| x^{n}\right) \log \frac{p\left(y^{n} \| x^{n}\right)}{\sum_{x^{n}} p\left(x^{n} \| y^{n-1}\right) p\left(y^{n} \| x^{n}\right)}+\sum_{y_{n}} p\left(y^{n} \| x^{n}\right) \\
&-\lambda_{x^{n}, y^{n-1}}+\sum_{i=0}^{n} \nu_{x^{i}, y^{n-1}}+\nu_{y^{n-1}} \\
&=-\sum_{y_{n}} p\left(y^{n} \| x^{n}\right) \log \frac{p\left(y^{n} \| x^{n}\right)}{e p\left(y^{n}\right)}-\lambda_{x^{n}, y^{n-1}}+\sum_{i=0}^{n} \nu_{x^{i}, y^{n-1}}+\nu_{y^{n-1}} \\
& \frac{\partial L}{\partial \gamma_{x^{i}, y^{i-1}}}=-\sum_{i=0}^{n} \nu_{x^{i}, y^{n-1}}
\end{aligned}
$$

Hence, the KKT conditions given in (21) become

$$
\begin{aligned}
& \sum_{y_{n}} p\left(y^{n} \| x^{n}\right) \log \frac{p\left(y^{n} \| x^{n}\right)}{e p\left(y^{n}\right)}=\nu_{y^{n-1}} \forall x^{n}, y^{n-1} \text { if } P\left(x^{n} \| y^{n-1}\right)>0, \\
& \sum_{y_{n}} p\left(y^{n} \| x^{n}\right) \log \frac{p\left(y^{n} \| x^{n}\right)}{e p\left(y^{n}\right)} \leq \nu_{y^{n-1}} \forall x^{n}, y^{n-1} \text { if } p\left(x^{n} \| y^{n-1}\right)=0,
\end{aligned}
$$

which is exactly (15). Now, to obtain (16) we use (24) and observe that

$$
\sum_{x^{n}} \sum_{y^{n-1}} p\left(x^{n} \| y^{n-1}\right) \sum_{y_{n}} p\left(y^{n} \| x^{n}\right) \log \frac{p\left(y^{n} \| x^{n}\right)}{e p\left(y^{n}\right)}=\sum_{X^{n}} \sum_{y^{n-1}} p\left(x^{n} \| y^{n-1}\right) \nu_{y^{n-1}} .
$$

Indeed, the LHS of (25) equals to $I\left(X^{n} \rightarrow Y^{n}\right)-1$. The RHS equals to $\sum_{y^{n-1}} \nu_{y^{n-1}}$, since $\sum_{x^{n}} p\left(x^{n} \| y^{n-1}\right)=1$ for all $y^{n-1}$ and, therefore, (25) implies that (16) holds. 
The next corollary is the main tool we use in this paper to prove that the feedback capacity and the non feedback capacity of a channel are equal.

Corollary 3: Let $P^{*}\left(x^{n} \| y^{n-1}\right)$ be a pmf that all its elements are positive and that achieves the maximum of $\max _{P\left(x^{n} \| y^{n-1}\right)} I\left(X^{n} \rightarrow Y^{n}\right)$, and let $P^{*}\left(y^{n}\right)$ be the output probability induced by $P^{*}\left(x^{n} \| y^{n-1}\right)$. If for any $n$ there exists an input probability distribution $P\left(x^{n}\right)$ such that

$$
p^{*}\left(y^{n}\right)=\sum_{x^{n}} p\left(y^{n} \| x^{n}\right) p\left(x^{n}\right),
$$

then the feedback capacity and the nonfeedback capacity are the same.

Proof: Note that the sufficient and necessary condition given in 15 depends only on the channel causal conditioning pmf $P\left(y^{n} \| x^{n}\right)$ and the output pmf $P\left(y^{n}\right)$. Furthermore, note that if (15) is satisfied then

$$
\sum_{y^{n}} p\left(y^{n} \| x^{n}\right) \log \frac{p\left(y^{n} \| x^{n}\right)}{e p\left(y^{n}\right)}=\sum_{y^{n-1}} \beta_{y^{n-1}}, \quad \forall x^{n},
$$

and since for the non-feedback case $p\left(y^{n} \| x^{n}\right)=p\left(y^{n} \mid x^{n}\right), \forall\left(x^{n}, y^{n}\right)$, we obtain

$$
\sum_{y^{n}} p\left(y^{n} \mid x^{n}\right) \log \frac{p\left(y^{n} \mid x^{n}\right)}{e p\left(y^{n}\right)}=\sum_{y^{n-1}} \beta_{y^{n-1}}, \quad \forall x^{n} .
$$

This means that the KKT conditions of $\max _{P\left(x^{n}\right)} I\left(X^{n} ; Y^{n}\right)$ are satisfied. Furthermore, the maximum value for both optimization problems is $\sum_{y^{n-1}} \beta_{y^{n-1}}+1$ and, therefore, they are equal.

\section{CAPACITY OF THE POST $(\alpha)$ CHANNEL WITH AND WITHOUT FEEDBACK}

Lemma 4 (Feedback capacity): The feedback capacity of the $\operatorname{POST}(\alpha)$ channel is the same as of the memoryless $Z$ channel with parameter $\alpha$, which is $C=-\log _{2} c$ where

$$
c=\left(1+\bar{\alpha} \alpha^{\frac{\alpha}{\bar{\alpha}}}\right)^{-1}
$$

The behavior of the capacity as a function of $\alpha$ is depicted in Fig. 3 .

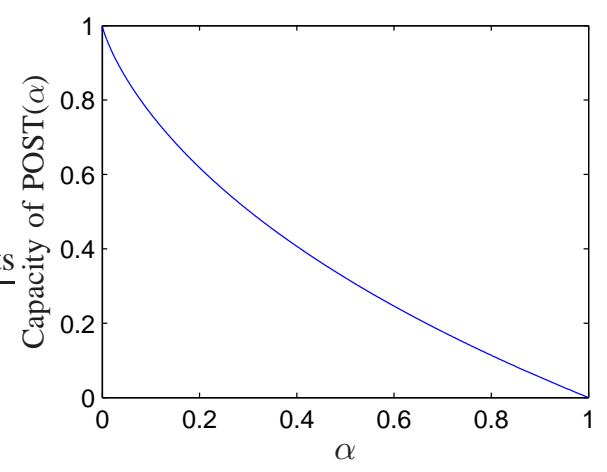

Fig. 3. The capacity of the $\operatorname{POST}(\alpha)$ channel with and without feedback. This is also the capacity of the $Z$ channel with parameter $\alpha$ 
Proof of the achievability and the capacity of $Z$ channel with parameter $\alpha$ : the achievability proof is trivial since both the encoder and the decoder know if the channel behaves as a $Z$ or an $S$ channel. The input probability that maximizes the mutual information, i.e. $\arg \max _{P_{X}} I(X ; Y)$, for the memoryless $Z$ channels with parameter $\alpha$ is

$$
P(x=1)=c \alpha^{\frac{\alpha}{\alpha}} \quad P(x=0)=c\left(1-\alpha^{\frac{1}{\alpha}}\right),
$$

where $c$ is a normalization coefficient and is given in (29). The output probability for the $Z$ channel with parameter $\alpha$ is

$$
\begin{aligned}
& P(y=1)=\bar{\alpha} P(x=1)=c \bar{\alpha} \alpha^{\frac{\alpha}{\alpha}} \\
& P(y=0)=1-c \bar{\alpha} \alpha^{\frac{\alpha}{\alpha}}=c .
\end{aligned}
$$

The capacity of the $Z$ channel with parameter $\alpha$ is

$$
C=\max _{P_{X}} I(X ; Y)=-\log _{2} c,
$$

which is also an achievable rate for the $\operatorname{POST}(\alpha)$ channel with feedback.

Proof of converse: The upper bound is given in the following set of equalities and inequalities:

$$
\begin{aligned}
C_{f b} & \stackrel{(a)}{=} \lim _{n \rightarrow \infty} \max _{P\left(x^{n} \| y^{n-1}\right)} \frac{1}{n} I\left(X^{n} \rightarrow Y^{n}\right) \\
& \stackrel{(b)}{=} \lim _{n \rightarrow \infty} \max _{P\left(x^{n}|| y^{n-1}\right)} \frac{1}{n} I\left(X^{n} \rightarrow Y^{n} \mid Y_{0}\right) \\
& =\lim _{n \rightarrow \infty} \max _{P\left(x^{n}|| y^{n-1}\right)} \frac{1}{n} \sum_{i=1}^{n} H\left(Y_{i} \mid Y^{i-1}\right)-H\left(Y_{i} \mid Y^{i-1}, X^{i}\right) \\
& \stackrel{(c)}{\leq} \lim _{n \rightarrow \infty} \max _{P\left(x^{n}|| y^{n-1}\right)} \frac{1}{n} \sum_{i=1}^{n} H\left(Y_{i} \mid Y_{i-1}\right)-H\left(Y_{i} \mid Y_{i-1}, X_{i}\right) \\
& =\lim _{n \rightarrow \infty} \max _{P\left(x^{n}|| y^{n-1}\right)} \sum_{i=1}^{n} P\left(y_{i}=0\right) I\left(X_{i} ; Y_{i} \mid y_{i-1}=0\right)+P\left(y_{i}=1\right) I\left(X_{i} ; Y_{i} \mid y_{i-1}=1\right) \\
& \stackrel{(d)}{=} \quad \lim _{n \rightarrow \infty} \max _{\left\{P\left(x_{i} \mid y_{i-1}\right)\right\}_{i \geq 1}} \sum_{i=1}^{n} P\left(y_{i}=0\right) I\left(X_{i} ; Y_{i} \mid y_{i-1}=0\right)+P\left(y_{i}=1\right) I\left(X_{i} ; Y_{i} \mid y_{i-1}=1\right) \\
& =\max _{P\left(x_{i} \mid y_{i-1}=0\right)} P\left(y_{i}=0\right) I\left(X_{i} ; Y_{i} \mid y_{i-1}=0\right)+\underset{P\left(x_{i} \mid y_{i-1}=1\right)}{\max } P\left(y_{i}=1\right) I\left(X_{i} ; Y_{i} \mid y_{i-1}=1\right), \text { for some } i \\
& \stackrel{(e)}{=} \quad-P\left(y_{i}=0\right) \log _{2} c-P\left(y_{i}=1\right) \log _{2} c, \\
& =-\log _{2} c,
\end{aligned}
$$

where (a) follows from the capacity formula given in [10], [19], (b) from the inequality $\mid I\left(X^{n} \rightarrow Y^{n}\right)-I\left(X^{n} \rightarrow\right.$ $\left.Y^{n} \mid S\right) \mid \leq H(S)$ [19, Lemma 4], (c) from the fact that conditioning reduces entropy and from the Markov chain $Y_{i}-\left(X_{i}, Y_{i-1}\right)-\left(X^{i-1}, Y^{i-2}\right)$, (d) from the fact that the set of causal conditioning $P\left(x^{n} \| y^{n-1}\right)$ is equivalent to the set of $\left\{P\left(x_{i} \mid x^{i-1}, y^{i-1}\right)\right\}$ and in this particular case it's enough to maximize only over $\left\{P\left(x_{i} \mid y_{i-1}\right)\right\}$ because of the objective. Finally, Step (e) follows from the capacity of the memoryless $Z$-channel with parameter $\alpha$. 
Note that the induced $Y_{i}$ is a Markov chain with transition probability $c \bar{\alpha} \alpha^{\frac{\alpha}{\bar{\alpha}}}$ (as $X_{i}$ depends on the past $X^{i-1}, Y^{i-1}$ only through $\left.Y_{i-1}\right)$. Now, we are interested in expressing the conditional pmf of the POST $(\alpha)$ channel recursively. This recursive formula will be used later to find an input distribution that does not utilize the feedback for the case of a POST Channel without feedback and achieves the same output distribution, namely, a Markov chain with transition probability $c \bar{\alpha} \alpha^{\frac{\alpha}{\bar{\alpha}}}$.

Table 1 presents the conditional pmf of a $\operatorname{POST}(\alpha)$ channel when $n=1$. Let us denote by $P_{n, 0}$ and $P_{n, 1}$ the

\begin{tabular}{|c|c|c|}
\hline$Y_{1}$ & 0 & 1 \\
\hline 0 & 1 & $\alpha$ \\
\hline 1 & 0 & $\bar{\alpha}$ \\
\hline
\end{tabular}

\begin{tabular}{|c|c|c|}
\hline$Y_{Y_{1}} X_{1}$ & 0 & 1 \\
\hline 0 & $\bar{\alpha}$ & 0 \\
\hline 1 & $\alpha$ & 1 \\
\hline
\end{tabular}

TABLE I

Conditional probabilities $P\left(Y_{1} \mid X_{1}, s_{0}=0\right)$ (on Left) And $P\left(Y_{1} \mid X_{1}, s_{0}=1\right)$ (on the Right) of the POST $(\alpha)$ Channel.

conditional matrices of the channel given the respective initial state, i.e., $s_{0}$ is 0 and 1 , respectively. Namely,

$$
\begin{aligned}
& P_{n, 0} \triangleq P\left(y^{n} \| x^{n}, s_{0}=0\right) \\
& P_{n, 1} \triangleq P\left(y^{n} \| x^{n}, s_{0}=1\right) .
\end{aligned}
$$

The columns of the matrices $P_{n, 0}$ and $P_{n, 1}$ are indexed by $x^{n}=\left(x_{1}, x_{2}, \ldots, x_{n}\right)$ and the rows by $y^{n}=\left(y_{1}, y_{2}, \ldots, y_{n}\right)$ arranged via lexicographical order, where $x_{1}$ and $y_{1}$ are the most significant bits and $x_{n}$ and $y_{n}$ are the least significant bits. For instance, the conditional probabilities $P\left(y_{1} \mid x_{1}, s_{0}=0\right)$ and $P\left(y_{1} \mid x_{1}, s_{0}=1\right)$ are given in Table I Hence, $P_{n, 0}$ and $P_{n, 1}$, for $n=1$, are given by

$$
P_{1,0}=\left[\begin{array}{cc}
1 & \alpha \\
0 & \bar{\alpha}
\end{array}\right] \quad P_{1,1}=\left[\begin{array}{cc}
\bar{\alpha} & 0 \\
\alpha & 1
\end{array}\right] \text {. }
$$

\begin{tabular}{|c|c|c|c|c|}
\hline$Y_{1} Y_{2} X_{1} X_{2}$ & 00 & 01 & 10 & 11 \\
\hline 00 & 1 & $\alpha$ & $\alpha$ & $\alpha^{2}$ \\
\hline 01 & 0 & $\bar{\alpha}$ & 0 & $\alpha \bar{\alpha}$ \\
\hline 10 & 0 & 0 & $\bar{\alpha}^{2}$ & 0 \\
\hline 11 & 0 & 0 & $\bar{\alpha} \alpha$ & $\bar{\alpha}$ \\
\hline
\end{tabular}

Table presents $P\left(y^{2} \| x^{2}, s_{0}=0\right)$ and the corresponding matrix $P_{2,0}$ is given in eq. (36).

TABLE II

Conditional PRoBABilities $P\left(Y^{2} \| X^{2}, s_{0}=0\right)$. 


$$
P_{2,0}=\left[\begin{array}{cccc}
1 & \alpha & \alpha & \alpha^{2} \\
0 & \bar{\alpha} & 0 & \alpha \bar{\alpha} \\
0 & 0 & \bar{\alpha}^{2} & 0 \\
0 & 0 & \bar{\alpha} \alpha & \alpha
\end{array}\right]
$$

From the channel definition, the following recursive relation holds

$$
P_{n, 0}=\left[\begin{array}{cc}
1 \cdot P_{n-1,0} & \alpha \cdot P_{n-1,0} \\
0 \cdot P_{n-1,1} & \bar{\alpha} \cdot P_{n-1,1}
\end{array}\right]
$$

and

$$
P_{n, 1}=\left[\begin{array}{cc}
\bar{\alpha} \cdot P_{n-1,0} & 0 \cdot P_{n-1,0} \\
\alpha \cdot P_{n-1,1} & 1 \cdot P_{n-1,1}
\end{array}\right]
$$

where $P_{0,0}=P_{0,1}=1$ (i.e., the one by one unit matrix).

Recall that the output process $\left\{Y_{i}\right\}_{i \geq 1}$ induced by the input that achieves the feedback capacity, is a binary symmetric Markov chain with transition probability $c \bar{\alpha} \alpha^{\frac{\alpha}{\alpha}}$, see (31). Let $p_{0}\left(y^{n}\right)$ and $p_{1}\left(y^{n}\right)$ denote the probability of $y^{n}$ given the initial state 0 and 1 , respectively. Hence, for $n=1$ we have

$$
\begin{aligned}
& p_{0}\left(y_{1}=0\right)=p_{1}\left(y_{1}=1\right)=c \\
& p_{0}\left(y_{1}=1\right)=p_{1}\left(y_{1}=0\right)=c \bar{\alpha} \alpha^{\frac{\alpha}{\alpha}} .
\end{aligned}
$$

For $n \geq 2$,

$$
p_{0}\left(y_{n} \mid y^{n-1}\right)=p_{1}\left(y_{n} \mid y^{n-1}\right)=p_{y_{n-1}}\left(y_{n}\right)
$$

Now, we present this Markov process in a recursive way. Recall that $P\left(y^{n}\right)$ is represented as a (column) probability vector of dimension $2^{n}$.

Lemma 5 (vector representation of a pmf of a symmetric Markov process): Let $Y^{n}$ be binary symmetric Markov with transition probability $\delta$. Let $P_{0}\left(y^{n}\right)$ and $P_{1}\left(y^{n}\right)$ be the vector pmf when the intial state is 0 and 1 , respectively. One can describe the vector pmf using the following recursive relation

$$
P_{0}\left(y^{n}\right)=\left[\begin{array}{c}
\bar{\delta} P_{0}\left(y^{n-1}\right) \\
\delta P_{1}\left(y^{n-1}\right)
\end{array}\right] \quad \text { and } \quad P_{1}\left(y^{n}\right)=\left[\begin{array}{c}
\delta P_{0}\left(y^{n-1}\right) \\
\bar{\delta} P_{1}\left(y^{n-1}\right)
\end{array}\right]
$$

where $P_{0}\left(y^{0}\right)=P_{1}\left(y^{0}\right)=1$, and $\left[\begin{array}{l}u \\ v\end{array}\right]$ denotes the column vector obtained by concatenating the two column vectors $u$ and $v$.

Proof: We prove this claim by induction. Note that, for $n=1$ (41) implies

$$
\begin{aligned}
& p_{0}\left(y_{1}=0\right)=p_{1}\left(y_{1}=1\right)=\bar{\delta} \\
& p_{0}\left(y_{1}=1\right)=p_{1}\left(y_{1}=0\right)=\delta .
\end{aligned}
$$


Now, we need to show that, regardless of the initial state, 40 holds. Assume $y_{1}=1$ and the initial state is 0 , then for any $y_{2}^{n}$

$$
\begin{aligned}
p_{0}\left(y_{n} \mid y^{n-1}\right) & =\frac{p_{0}\left(y^{n}\right)}{p_{0}\left(y^{n-1}\right)} \\
& =\frac{p_{0}\left(1, y_{2}^{n}\right)}{p_{0}\left(1, y_{2}^{n-1}\right)} \\
& =\frac{\delta p_{1}\left(y_{2}^{n}\right)}{\delta p_{1}\left(y_{2}^{n-1}\right)} \\
& =\frac{p_{1}\left(y_{2}^{n}\right)}{p_{1}\left(y_{2}^{n-1}\right)} \\
& =p_{1}\left(y_{n} \mid y_{2}^{n-1}\right) .
\end{aligned}
$$

In a similar way we obtain for any $y_{2}^{n}$ and any initial state

$$
p_{0}\left(y_{n} \mid y^{n-1}\right)=p_{1}\left(y_{n} \mid y^{n-1}\right)=p_{y_{1}}\left(y_{n} \mid y_{2}^{n-1}\right)
$$

By repeating the procedure in (43) $i$ times, we obtain that for $1 \leq i<n$ and $\forall y_{2}^{n}$

$$
p_{0}\left(y_{n} \mid y^{n-1}\right)=p_{1}\left(y_{n} \mid y^{n-1}\right)=p_{y_{i}}\left(y_{n} \mid y_{i+1}^{n-1}\right)
$$

Now, note that choosing $i=n-1$ we obtain (40), which means that the process is indeed Markov with transition probability $\delta$.

The following is our first main result:

Theorem 6: Feedback does not increase the capacity of the POST $(\alpha)$ channel.

Proof: According to Corollary 3, in order to show that the nonfeedback capacity equals the feedback capacity, it suffices to show that there exists an input pmf $P\left(x^{n}\right)$ that induces the optimal $P^{*}\left(y^{n}\right)$ of the feedback case, which is the binary symmetric Markov chain with transition probability $c \bar{\alpha} \alpha^{\frac{\alpha}{\alpha}}$.

We will now find such a pmf by calculating $P_{1}\left(x^{n}\right)=P_{n, 1}^{-1} P_{1}\left(y^{n}\right)$ and $P_{0}\left(x^{n}\right)=P_{n, 0}^{-1} P_{0}\left(y^{n}\right)$ and verifying that $P_{0}\left(x^{n}\right)$ and $P_{1}\left(x^{n}\right)$ are indeed a valid pmf. Recall that

$$
\left[\begin{array}{ll}
A & B \\
0 & D
\end{array}\right]^{-1}=\left[\begin{array}{cc}
A^{-1} & -A^{-1} B D^{-1} \\
0 & D^{-1}
\end{array}\right] .
$$

Hence,

$$
\begin{aligned}
& P_{n, 0}^{-1}=\left[\begin{array}{cc}
1 \cdot P_{n-1,0} & \alpha \cdot P_{n-1,0} \\
0 \cdot P_{n-1,1} & \bar{\alpha} \cdot P_{n-1,1}
\end{array}\right]^{-1}=\left[\begin{array}{cc}
P_{n-1,0}^{-1} & -\frac{\alpha}{\bar{\alpha}} P_{n-1,1}^{-1} \\
0 & \frac{1}{\bar{\alpha}} P_{n-1,1}^{-1}
\end{array}\right] \\
& P_{n, 1}^{-1}=\left[\begin{array}{ll}
\bar{\alpha} \cdot P_{n-1,0} & 0 \cdot P_{n-1,0} \\
\alpha \cdot P_{n-1,1} & 1 \cdot P_{n-1,1}
\end{array}\right]^{-1}=\left[\begin{array}{cc}
\frac{1}{\bar{\alpha}} P_{n-1,0}^{-1} & 0 \\
-\frac{\alpha}{\bar{\alpha}} P_{n-1,0}^{-1} & P_{n-1,1}^{-1}
\end{array}\right]
\end{aligned}
$$

Now we compute $P_{1}\left(x^{n}\right)$ and $P_{0}\left(x^{n}\right)$. 


$$
\begin{aligned}
P_{0}\left(x^{n}\right) & =P_{n, 0}^{-1} P_{0}\left(y^{n}\right) \\
& =\left[\begin{array}{cc}
P_{n-1,0}^{-1} & -\frac{\alpha}{\bar{\alpha}} P_{n-1,1}^{-1} \\
0 & \frac{1}{\bar{\alpha}} P_{n-1,1}^{-1}
\end{array}\right]\left[\begin{array}{c}
c P_{0}\left(y^{n-1}\right) \\
c \bar{\alpha} \alpha^{\frac{\alpha}{\bar{\alpha}}} P_{1}\left(y^{n-1}\right)
\end{array}\right] \\
& =c\left[\begin{array}{c}
P_{0}\left(x^{n-1}\right)-\alpha^{\frac{1}{\bar{\alpha}}} P_{1}\left(x^{n-1}\right) \\
\alpha^{\frac{\alpha}{\alpha}} P_{1}\left(x^{n-1}\right)
\end{array}\right], \\
P_{1}\left(x^{n}\right)= & P_{n, 1}^{-1} P_{1}\left(y^{n}\right) \\
= & {\left[\begin{array}{c}
\frac{1}{\bar{\alpha}} P_{n-1,0}^{-1} \\
-\frac{\alpha}{\bar{\alpha}} P_{n-1,0}^{-1}
\end{array}\right]\left[\begin{array}{c}
c \bar{\alpha} \alpha^{\frac{\alpha}{\alpha}} P_{0}\left(y^{n-1}\right) \\
c P_{1}\left(y^{n-1}\right)
\end{array}\right] } \\
= & {\left[\begin{array}{c}
\alpha^{\frac{\alpha}{\alpha}} P_{0}\left(x^{n-1}\right) \\
P_{1}\left(x^{n-1}\right)-\alpha^{\frac{1}{\alpha}} P_{0}\left(x^{n-1}\right)
\end{array}\right], }
\end{aligned}
$$

where $P_{0}\left(x^{0}\right)=P_{1}\left(x^{0}\right)=1$.

Now, we need to show that the probability expressions are valid, namely, nonnegative and sum to 1 . The fact that they sum to 1 can be seen from the recursion immediately by verifying that $c\left(\alpha^{\frac{\alpha}{\alpha}}+1-\alpha^{\frac{1}{\alpha}}\right)=1$.

In order to show the nonnegativity we need to show that

$$
\begin{aligned}
& P_{0}\left(x^{n-1}\right)-\alpha^{\frac{1}{\alpha}} P_{1}\left(x^{n-1}\right) \geq 0 \\
& P_{1}\left(x^{n-1}\right)-\alpha^{\frac{1}{\bar{\alpha}}} P_{0}\left(x^{n-1}\right) \geq 0 .
\end{aligned}
$$

For $n=1$ this is true since $\alpha^{\frac{1}{\alpha}} \leq 1$ for $0 \leq \alpha \leq 1$ (see Lemma 17 in the appendix). The following lemma (Lemma 7) states that if (51) holds for $n-1$ it also holds for $n$ and, by induction, we conclude that (51) holds for all $n$.

Lemma 7: There exists a $1 \leq \beta \leq \alpha^{-\frac{1}{\alpha}}$, for which the condition

$$
\begin{aligned}
& \beta P_{1}\left(x^{n-1}\right) \geq P_{0}\left(x^{n-1}\right), \forall x^{n-1}, \\
& \beta P_{0}\left(x^{n-1}\right) \geq P_{1}\left(x^{n-1}\right), \forall x^{n-1},
\end{aligned}
$$

implies

$$
\begin{aligned}
& \beta P_{1}\left(x^{n}\right) \geq P_{0}\left(x^{n}\right), \forall x^{n}, \\
& \beta P_{0}\left(x^{n}\right) \geq P_{1}\left(x^{n}\right), \forall x^{n} .
\end{aligned}
$$

\section{Proof:}

Let's assume that (52) holds and we need to show that (53) holds, which is equivalent to showing the following four inequalities:

$$
\begin{gathered}
\beta\left(c P_{0}\left(x^{n-1}\right)-c \alpha^{\frac{1}{\alpha}} P_{1}\left(x^{n-1}\right)\right) \geq c \alpha^{\frac{\alpha}{\bar{\alpha}}} P_{0}\left(x^{n-1}\right), \\
\beta c \alpha^{\frac{\alpha}{\alpha}} P_{1}\left(x^{n-1}\right) \geq c P_{1}\left(x^{n-1}\right)-c \alpha^{\frac{1}{\alpha}} P_{0}\left(x^{n-1}\right),
\end{gathered}
$$




$$
\begin{gathered}
\beta c \alpha^{\frac{\alpha}{\alpha}} P_{0}\left(x^{n-1}\right) \geq c P_{0}\left(x^{n-1}\right)-c \alpha^{\frac{1}{\alpha}} P_{1}\left(x^{n-1}\right), \\
\beta\left(c P_{1}\left(x^{n-1}\right)-c \alpha^{\frac{1}{\alpha}} P_{0}\left(x^{n-1}\right)\right) \geq c \alpha^{\frac{\alpha}{\alpha}} P_{1}\left(x^{n-1}\right) .
\end{gathered}
$$

Because of the symmetry it suffices to show that (54) and (55) hold. We start by showing that (54) holds. The inequality (54) is equivalent to

$$
\beta\left(P_{0}\left(x^{n-1}\right)-\alpha^{\frac{1}{\alpha}} P_{1}\left(x^{n-1}\right)\right) \geq \alpha^{\frac{\alpha}{\alpha}} P_{0}\left(x^{n-1}\right)
$$

which can be further written as

$$
P_{0}\left(x^{n-1}\right)\left(\beta-\alpha^{\frac{\alpha}{\bar{\alpha}}}\right) \geq \beta \alpha^{\frac{1}{\bar{\alpha}}} P_{1}\left(x^{n-1}\right)
$$

and as

$$
P_{0}\left(x^{n-1}\right) \frac{\beta-\alpha^{\frac{\alpha}{\bar{\alpha}}}}{\beta \alpha^{\frac{1}{\bar{\alpha}}}} \geq P_{1}\left(x^{n-1}\right) .
$$

This is true using the induction assumption in 52 if

$$
\frac{\beta-\alpha^{\frac{\alpha}{\bar{\alpha}}}}{\beta \alpha^{\frac{1}{\alpha}}} \geq \beta
$$

Equivalently

$$
\begin{gathered}
\alpha^{\frac{1}{\alpha}} \beta^{2}-\beta+\alpha^{\frac{\alpha}{\alpha}} \leq 0 . \\
\frac{1-\sqrt{1-4 \alpha^{\frac{\alpha+1}{\alpha}}}}{2 \alpha^{\frac{1}{\alpha}}} \leq \beta \leq \frac{1+\sqrt{1-4 \alpha^{\frac{\alpha+1}{\alpha}}}}{2 \alpha^{\frac{1}{\alpha}}}
\end{gathered}
$$

where the condition $4 \alpha^{\frac{\alpha+1}{\alpha}} \leq 1$ can be verified to hold for all $0 \leq \alpha \leq 1$, as shown in Lemma 18 in the appendix.

Now let's consider inequality 55. We need to show that

$$
\beta \alpha^{\frac{\alpha}{\alpha}} P_{1}\left(x^{n-1}\right) \geq P_{1}\left(x^{n-1}\right)-\alpha^{\frac{1}{\alpha}} P_{0}\left(x^{n-1}\right)
$$

or, equivalently,

$$
P_{0}\left(x^{n-1}\right) \geq \frac{1-\beta \alpha^{\frac{\alpha}{\bar{\alpha}}}}{\alpha^{\frac{1}{\alpha}}} P_{1}\left(x^{n-1}\right) .
$$

This is true using the induction assumption in (52) if

$$
\frac{1}{\beta} \geq \frac{1-\beta \alpha^{\frac{\alpha}{\bar{\alpha}}}}{\alpha^{\frac{1}{\bar{\alpha}}}}
$$

and equivalently

$$
\alpha^{\frac{\alpha}{\bar{\alpha}}} \beta^{2}-\beta+\alpha^{\frac{1}{\bar{\alpha}}} \geq 0
$$

This holds if

$$
\beta \geq \frac{1+\sqrt{1-4 \alpha^{\frac{\alpha+1}{\bar{\alpha}}}}}{2 \alpha^{\frac{\alpha}{\bar{\alpha}}}} .
$$

Combining 63 with 68 we obtain that there exists a $\beta$ in the interval

$$
\frac{1+\sqrt{1-4 \alpha^{\frac{\alpha+1}{\bar{\alpha}}}}}{2 \alpha^{\frac{\alpha}{\bar{\alpha}}}} \leq \beta \leq \frac{1+\sqrt{1-4 \alpha^{\frac{\alpha+1}{\bar{\alpha}}}}}{2 \alpha^{\frac{1}{\alpha}}}
$$


that satisfies the lemma. Note that the interval is nonempty since for $0 \leq \alpha \leq 1, \alpha^{\frac{\alpha}{\alpha}} \geq \alpha^{\frac{1}{\alpha}}$. Finally, note that since $\frac{1+\sqrt{1-4 \alpha^{\frac{\alpha+1}{\alpha}}}}{2} \leq 1$ we obtain that $\beta \leq \alpha^{-\frac{1}{\alpha}}$. Furthermore, it is shown in Lemma 19 in the appendix that $\frac{1+\sqrt{1-4 \alpha^{\frac{\alpha+1}{\alpha}}}}{2 \alpha^{\frac{\alpha}{\alpha}}} \geq 1$ for any $0 \leq \alpha \leq 1$, and, therefore, also $\beta \geq 1$.

In the proof that feedback does not increase the capacity of the $\operatorname{POST}(\alpha)$ channel (Theorem 6 ) we actually found a recursive formula of the input distribution that achieves the capacity (see (49)-(50). It is interesting that the input distribution is not a Markov distribution and has an infinite memory but it induces a simple Markov distribution at the output of the channel. In addition, as shown in the following lemma, the input distribution is stationary.

Lemma 8 (Stationarity of the input distribution): The probabilities $P_{0}\left(x^{n}\right)$ and $P_{1}\left(x^{n}\right)$ that achieves the capacity of the $\operatorname{POST}(\alpha)$ channel (i.e., achieve the maximum in (11) when the mutual information is conditioned, respectively, on the initial state being 0 and 1), and are specified in (49)-(50), are stationary, namely, for any $n$ and $i<n$, and for any $x^{n}$

$$
p_{0}\left(x^{i}\right)=p_{0}\left(x_{n-i+1}^{n}\right), \quad p_{1}\left(x^{i}\right)=p_{1}\left(x_{n-i+1}^{n}\right)
$$

Proof: We prove first the case $i=1$ and then generalize it to any $i$. For any $n$ and $i=1$ we have $p_{0}\left(x_{n}\right)=$ $\sum_{x^{n-1}} p_{0}\left(x^{n}\right)$. Note that applying this relation to (49) we obtain

$$
P_{0}\left(x_{n}\right)=c\left[\begin{array}{c}
1-\alpha^{\frac{1}{\alpha}} \\
\alpha^{\frac{\alpha}{\alpha}}
\end{array}\right],
$$

for all $n$. We can now generalize it to any $i$ by summing over $x^{n-i}$ terms and we have

$$
P_{0}\left(x_{n-i+1}^{n}\right)=c\left[\begin{array}{c}
P_{0}\left(x_{n-i+1}^{n-1}\right)-\alpha^{\frac{1}{\alpha}} P_{1}\left(x_{n-i+1}^{n-1}\right) \\
\alpha^{\frac{\alpha}{\alpha}} P_{1}\left(x_{n-i+1}^{n-1}\right)
\end{array}\right] .
$$

Hence, this is the same iterative equation we have for $P_{0}\left(x^{i}\right)$ in 496, and since $P_{0}\left(x_{1}\right)=P_{0}\left(x_{n-i+1}\right)$ we obtain the same vector probability for all $n$. Similar proof holds $P_{1}\left(x^{n}\right)$.

\section{BINARY POST $(a, b)$ CHANNEL}

In this section, we extend the scope and study the capacity of what we refer to as the $\operatorname{POST}(a, b)$, which is a generalization of $\operatorname{POST}(\alpha)$ channel. The $\operatorname{POST}(a, b)$ channel has two states and in each state there is a binary channel with respective parameters $(a, b)$. We develop, using similar analyses as for the $\operatorname{POST}(\alpha)$ channel, simple conditions on $a, b$ for which, if satisfied, feedback does not increase capacity. We then show that the conditions are satisfied for all parameter values $(a, b) \in[0,1] \times[0,1]$.

\section{A. Definition of the POST $(a, b)$ channel}

Consider the POST channel depicted in Fig. 4 with the following behavior. When $y_{i-1}=0$, then the channel behaves as a binary channel with transition matrix

$$
\left[\begin{array}{ll}
a & \bar{b} \\
\bar{a} & b
\end{array}\right]
$$


and when $y_{i-1}=1$ then it behaves as a binary channel with the transition matrix

$$
\left[\begin{array}{ll}
b & \bar{a} \\
\bar{b} & a
\end{array}\right] \text {. }
$$

We refer to this channel as the $\operatorname{POST}(a, b)$ channel. $\operatorname{POST}(\alpha)$ is a special case of $\operatorname{POST}(a, b)$, where $a=1$ and
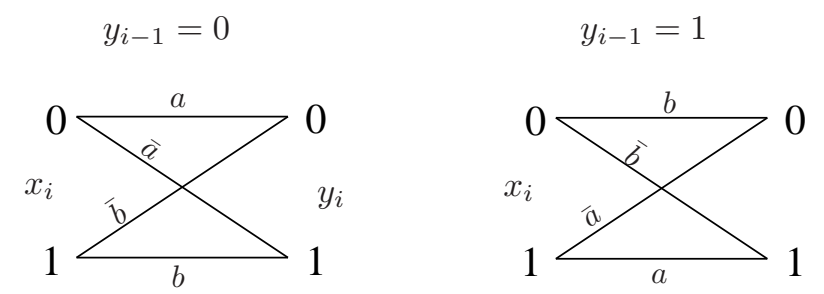

Fig. 4. $\operatorname{POST}(a, b)$ channel. If $y_{i-1}=0$ then the channel behaves as DMC with parameters $(a, b)$ and if $y_{i-1}=1$ then the channel behaves as DMC with parameters $(b, a)$.

$b=\bar{\alpha}$.

Without loss of generality, we assume throughout that $a+b-1>0$. It is easy to see that in the case where $a+b-1=0$ or, equivalently, where $a=\bar{b}$, the capacity is simply 0 . Additionally, if $a+b-1<0$ then $\bar{a}+\bar{b}>1$; hence by relabeling the inputs $(0 \leftrightarrow 1)$ we obtain a new channel (with parameter $a^{\prime}, b^{\prime}$ rather than $\left.a, b\right)$ where $a^{\prime}=\bar{a}$ and $b^{\prime}=\bar{b}$ and we have $a^{\prime}+b^{\prime}-1>0$.

\section{B. Capacity of the POST $(a, b)$ channel with and without feedback}

Before considering the $\operatorname{POST}(a, b)$ let us first consider the binary DMC with parameters $(a, b)$. The capacity of the binary DMC with parameters $(a, b)$ was derived by Ash in [22, Ex 3.7] by applying [22, Theorem 3.3.3] and is given by

$$
C=\log \left[2^{\frac{\bar{a} H_{b}(b)-b H_{b}(a)}{a+b-1}}+2^{\frac{\bar{b} H_{b}(a)-a H_{b}(b)}{a+b-1}}\right] .
$$

The capacity achieving input distribution is

$$
\begin{aligned}
& P(x=0)=c_{0}\left(b 2^{\frac{H(b)}{a+b-1}}-\bar{b} 2^{\frac{H(a)}{a+b-1}}\right), \\
& P(x=1)=c_{0}\left(-\bar{a} 2^{\frac{H(b)}{a+b-1}}+a 2^{\frac{H(a)}{a+b-1}}\right),
\end{aligned}
$$

where $c_{0}$ is a normalizing coefficient so that the sum $P(x=0)+P(x=1)$ is equal to 1 . The induced output distribution is

$$
\begin{aligned}
& P(y=0)=c_{0}(a b-\bar{a} \bar{b}) 2^{\frac{H(b)}{a+b-1}} \\
& P(y=1)=c_{0}(a b-\bar{a} \bar{b}) 2^{\frac{H(a)}{a+b-1}}
\end{aligned}
$$

Lemma 9 (Feedback capacity of $\operatorname{POST}(a, b))$ : The feedback capacity of the $\operatorname{POST}(a, b)$ channel is the same as of the memoryless DMC with parameters $(a, b)$, which is given in (75). 
The proof follows the same arguments as the proof of the feedback capacity of POST $(\alpha)$ in Lemma 4 and is, therefore, omitted. The behavior of the capacity as a function of $(a, b)$ is depicted in Fig. 5 .

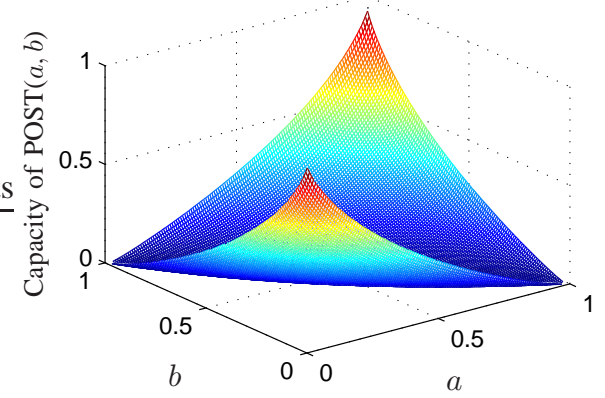

Fig. 5. The capacity of the $\operatorname{POST}(a, b)$ channel with and without feedback. This is also the capacity of the binary DMC with parameters $(a, b)$

We now present sufficient conditions on $a, b$ implying that feedback does not increase the capacity of the $\operatorname{POST}(a, b)$ channel. That these conditions are indeed sufficient we establish in the next subsection. Define the following intervals:

$$
\begin{aligned}
& \mathcal{L}_{1}=\left\{\max \left(\frac{\bar{a}}{\bar{b}} \gamma, \frac{\gamma(\bar{a}+b)-\sqrt{\gamma^{2}(\bar{a}+b)^{2}-4 a \bar{b}}}{2 \bar{b}}\right) \leq \beta \leq \frac{\gamma(\bar{a}+b)+\sqrt{\gamma^{2}(\bar{a}+b)^{2}-4 a \bar{b}}}{2 \bar{b}} .\right\} \\
& \mathcal{L}_{2}=\left\{\frac{(a+\bar{b})+\sqrt{(a+\bar{b})^{2}-4 \bar{a} b \gamma^{2}}}{2 b \gamma} \leq \beta \leq \frac{\bar{a}}{\bar{b}} \gamma\right\} \\
& \mathcal{L}_{3}=\left\{\beta \leq \min \left(\frac{\bar{a}}{\bar{b}} \gamma, \frac{(a+\bar{b})-\sqrt{(a+\bar{b})^{2}-4 \bar{a} b \gamma^{2}}}{2 b \gamma}\right)\right\} \\
& \mathcal{L}_{4}=\left\{\beta \leq \min \left(\frac{b \gamma}{a}, \frac{\gamma(\bar{a}+b)-\sqrt{\gamma^{2}(\bar{a}+b)^{2}-4 a \bar{b}}}{2 a}\right)\right\} \\
& \mathcal{L}_{5}=\left\{\frac{\gamma(\bar{a}+b)+\sqrt{\gamma^{2}(\bar{a}+b)^{2}-4 a \bar{b}}}{2 a} \leq \beta \leq \frac{b \gamma}{a}\right\} \\
& \mathcal{L}_{6}=\left\{\max \left(\frac{b \gamma}{a}, \frac{(a+\bar{b})-\sqrt{(a+\bar{b})^{2}-4 \bar{a} b \gamma^{2}}}{2 \bar{a} \gamma}\right) \leq \beta \leq \frac{(a+\bar{b})+\sqrt{(a+\bar{b})^{2}-4 \bar{a} b \gamma^{2}}}{2 \bar{a} \gamma}\right\},
\end{aligned}
$$

where $\gamma$ is defined as

$$
\gamma=2^{\frac{H(b)-H(a)}{a+b-1}} .
$$

In addition, let

$$
\mathcal{L}_{0}=\left\{1 \leq \beta \leq \min \left(\frac{a}{\bar{a} \gamma}, \frac{b \gamma}{\bar{b}}\right)\right\}
$$

Lemma 10 (conditions to determine that feedback does not increase capacity of the $\operatorname{POST}(a, b)$ ): If the intersections of the intervals $\mathcal{L}_{1} \cup \mathcal{L}_{2} \cup \mathcal{L}_{3}$ with $\mathcal{L}_{4} \cup \mathcal{L}_{5} \cup \mathcal{L}_{6}$ and $\mathcal{L}_{0}$ is nonempty then feedback does not increase the capacity of the $\operatorname{POST}(a, b)$ channel.

Lemma 11: The condition in Lemma 10 holds for all POST channel parameters $(a, b)$. Thus, feedback does not increase capacity of $\operatorname{POST}(a, b)$. 
Proof: It suffices to show that the either the interval $\left(\mathcal{L}_{1} \cap \mathcal{L}_{5} \cap \mathcal{L}_{0}\right)$ or the interval $\left(\mathcal{L}_{2} \cap L_{6} \cap \mathcal{L}_{0}\right)$ is non empty. First we claim that the expression in the square roots of (79) are nonnegative, i.e.,

$$
\begin{aligned}
& \gamma^{2}(\bar{a}+b)^{2}-4 a \bar{b} \geq 0 \\
& (a+\bar{b})^{2}-4 \bar{a} b \gamma^{2} \geq 0
\end{aligned}
$$

as shown in Appendix $\overline{\mathrm{D}-\mathrm{B}}$ (Note that the second inequality follows from the first by switching between $a$ and $b$ ).

Recall that $a+b-1 \geq 0$ which implies $a \geq \bar{b}$ and $\bar{a} \leq b$. In addition, assume that $\bar{a} a \leq b \bar{b}$, and we show in Appendix $\overline{\mathrm{D}-\mathrm{A}}$ that together with $a \geq \bar{b}$ it implies that $a \geq b$. We now prove that if $\bar{a} a \leq b \bar{b}$, then $\left(\mathcal{L}_{1} \cap \mathcal{L}_{5} \cap \mathcal{L}_{0}\right)$ is nonempty. (Similarly, one can show that if $\bar{a} a \geq b \bar{b},\left(\mathcal{L}_{2} \cap L_{6} \cap \mathcal{L}_{0}\right)$ is non empty.) We first want to claim that the intersection $\left(\mathcal{L}_{1} \cap \mathcal{L}_{5}\right)$ is nonempty. Indeed, the lower bound of $\mathcal{L}_{5}$ is smaller than the upper bound of $\mathcal{L}_{1}$ since $\frac{1}{2 a} \leq \frac{1}{2 b}$. The upper bound of $\mathcal{L}_{5}$ is larger than the lower bound of $\mathcal{L}_{1}$ since $\frac{b \gamma}{a} \geq \frac{\bar{a} \gamma}{b}$ because $\bar{a} a \leq b \bar{b}$, and because,

$$
\frac{\gamma(\bar{a}+b)-\sqrt{\gamma^{2}(\bar{a}+b)^{2}-4 a \bar{b}}}{2 \bar{b}} \leq \frac{b \gamma}{a},
$$

where the inequality follows the fact that the LHS is less or equal to 1, as shown in Appendix D-C, and the RHS is great or equal to 1 , as show in Appendix D-D.

Now we need to show that the intersection $\left(\mathcal{L}_{1} \cap \mathcal{L}_{5}\right)$ with $\mathcal{L}_{0}$ is still non empty. We will first show that $\min \left\{\frac{a}{\bar{a} \gamma}, \frac{b \gamma}{b}\right\} \geq \frac{b \gamma}{a}$. Recall that $a \geq \bar{b}$ hence $\frac{b \gamma}{b} \geq \frac{b \gamma}{a}$. In addition $\gamma^{2} \leq \frac{a^{2}}{b \bar{a}}$ as shown in Appendix D-E Now we want to show that the lower bound of $\mathcal{L}_{0}$ which is 1 is not larger than the upper bound of the intersection $\left(\mathcal{L}_{1} \cap \mathcal{L}_{5}\right)$. First we claim that the upper bound of $\mathcal{L}_{5}$ is larger than 1, i.e., $\frac{b \gamma}{a} \geq 1$ for $a \geq \bar{b}$, as shown in Appendix D-D, Finally, we need to show that the upper bound of $\mathcal{L}_{1}$ is larger than 1, i.e.,

$$
\frac{\gamma(\bar{a}+b)}{2 \bar{b}} \geq 1
$$

as shown in Appendix D-F

\section{Deriving the sufficient conditions of Lemma 10}

Proof of Lemma 10. Let $P_{n, 0}$ and $P_{n, 1}$ be defined as in 34]. Following the channel definition we have

$$
P_{n, 0}=\left[\begin{array}{ll}
a \cdot P_{n-1,0} & \bar{b} \cdot P_{n-1,0} \\
\bar{a} \cdot P_{n-1,1} & b \cdot P_{n-1,1}
\end{array}\right]
$$

and

$$
P_{n, 1}=\left[\begin{array}{ll}
b \cdot P_{n-1,0} & \bar{a} \cdot P_{n-1,0} \\
\bar{b} \cdot P_{n-1,1} & a \cdot P_{n-1,1}
\end{array}\right]
$$

where $P_{0,0}=P_{0,1}=1$. Using the identity

$$
\left[\begin{array}{ll}
\mathbf{A} & \mathbf{B} \\
\mathbf{C} & \mathbf{D}
\end{array}\right]^{-1}=\left[\begin{array}{cc}
\mathbf{A}^{-1}+\mathbf{A}^{-1} \mathbf{B}\left(\mathbf{D}-\mathbf{C A}^{-1} \mathbf{B}\right)^{-1} \mathbf{C A}^{-1} & -\mathbf{A}^{-1} \mathbf{B}\left(\mathbf{D}-\mathbf{C A}^{-1} \mathbf{B}\right)^{-1} \\
-\left(\mathbf{D}-\mathbf{C A}^{-1} \mathbf{B}\right)^{-1} \mathbf{C A}^{-1} & \left(\mathbf{D}-\mathbf{C A}^{-1} \mathbf{B}\right)^{-1}
\end{array}\right]
$$


we obtain

$$
\begin{aligned}
& P_{n, 0}^{-1}=\left[\begin{array}{cc}
\frac{b}{b a-\bar{a} b} P_{0}^{-1} & -\frac{\bar{b}}{b a-\bar{a} b} P_{1}^{-1} \\
-\frac{\bar{a}}{b a-\bar{a} b} P_{0}^{-1} & \frac{a}{b a-\bar{a} \bar{a}} P_{1}^{-1}
\end{array}\right] \\
& P_{n, 1}^{-1}=\left[\begin{array}{cc}
\frac{a}{b a-\bar{a} b} P_{0}^{-1} & -\frac{\bar{a}}{b a-\bar{a} b} P_{1}^{-1} \\
-\frac{\bar{b}}{b a-\bar{a} b} P_{0}^{-1} & \frac{b}{b a-\bar{a} b} P_{1}^{-1}
\end{array}\right]
\end{aligned}
$$

Now we compute $P_{1}\left(x^{n}\right)$ and $P_{0}\left(x^{n}\right)$

$$
\begin{aligned}
P_{0}\left(x^{n}\right) & =P_{n, 0}^{-1} P_{0}\left(y^{n}\right) \\
& =\frac{1}{a+b-1}\left[\begin{array}{cc}
b P_{0}^{-1} & -\bar{b} P_{1}^{-1} \\
-\bar{a} P_{0}^{-1} & a P_{1}^{-1}
\end{array}\right]\left[\begin{array}{c}
2^{\frac{H(b)}{a+b-1}} P_{0}\left(y^{n-1}\right) \\
2^{\frac{H(a)}{a+b-1}} P_{1}\left(y^{n-1}\right)
\end{array}\right] \frac{1}{2^{\frac{H(b)}{a+b-1}}+2^{\frac{H(a)}{a+b-1}}} \\
& =\frac{1}{(a+b-1)\left(2^{\frac{H(b)}{a+b-1}}+2^{\frac{H(a)}{a+b-1}}\right)}\left[\begin{array}{c}
b 2^{\frac{H(b)}{a+b-1}} P_{0}\left(x^{n-1}\right)-\bar{b} 2^{\frac{H(a)}{a+b-1}} P_{1}\left(x^{n-1}\right) \\
-\bar{a} 2^{\frac{H(b)}{a+b-1}} P_{0}\left(x^{n-1}\right)+a 2^{\frac{H(a)}{a+b-1}} P_{1}\left(x^{n-1}\right)
\end{array}\right], \\
P_{1}\left(x^{n}\right) & =P_{n, 1}^{-1} P_{1}\left(y^{n}\right) \\
& =\frac{1}{(a+b-1)\left(2^{\frac{H(b)}{a+b-1}}+2^{\frac{H(a)}{a+b-1}}\right)}\left[\begin{array}{c}
a 2^{\frac{H(a)}{a+b-1}} P_{0}\left(x^{n-1}\right)-\bar{a} 2^{\frac{H(b)}{a+b-1}} P_{1}\left(x^{n-1}\right) \\
-\bar{b} 2^{\frac{H(a)}{a+b-1}} P_{0}\left(x^{n-1}\right)+b 2^{\frac{H(b)}{a+b-1}} P_{1}\left(x^{n-1}\right)
\end{array}\right],
\end{aligned}
$$

where $P_{0}\left(x^{0}\right)=P_{1}\left(x^{0}\right)=1$. We can rewrite $P_{0}\left(x^{n}\right)$ and $P_{1}\left(x^{n}\right)$ follows:

$$
\begin{aligned}
& P_{0}\left(x^{n}\right)=\frac{1}{(a+b-1)(\gamma+1)}\left[\begin{array}{c}
b \gamma P_{0}\left(x^{n-1}\right)-\bar{b} P_{1}\left(x^{n-1}\right) \\
-\bar{a} \gamma P_{0}\left(x^{n-1}\right)+a P_{1}\left(x^{n-1}\right)
\end{array}\right], \\
& P_{1}\left(x^{n}\right)=\frac{1}{(a+b-1)(\gamma+1)}\left[\begin{array}{c}
a P_{0}\left(x^{n-1}\right)-\bar{a} \gamma P_{1}\left(x^{n-1}\right) \\
-\bar{b} P_{0}\left(x^{n-1}\right)+b \gamma P_{1}\left(x^{n-1}\right)
\end{array}\right] .
\end{aligned}
$$

We need to show that indeed the probability expressions are valid, namely nonnegative and sum to 1 . Showing the non-negativity of each of the terms in the above expression is equivalent to showing $\forall n \geq 1$ and for all $x^{n-1}$,

$$
\begin{aligned}
& \min \left\{\frac{a}{\bar{a} \gamma}, \frac{b \gamma}{\bar{b}}\right\} P_{0}\left(x^{n-1}\right) \geq P_{1}\left(x^{n-1}\right) \\
& \min \left\{\frac{a}{\bar{a} \gamma}, \frac{b \gamma}{\bar{b}}\right\} P_{1}\left(x^{n-1}\right) \geq P_{0}\left(x^{n-1}\right)
\end{aligned}
$$

For $n=1$ this follows from the fact that $\min \left\{\frac{a}{\bar{a} \gamma}, \frac{b \gamma}{b}\right\} \geq 1$ which is proved in Appendix D-G To prove for $n \geq 1$ we use the following lemma, whose proof appears in Appendix C

Lemma 12: If the condition in Lemma 10 holds then there exists, $1 \leq \beta \leq \min \left\{\frac{a}{\bar{a} \gamma}, \frac{b \gamma}{b}\right\}$ such that $\forall n$, the inequalities

$$
\begin{aligned}
& \beta P_{1}\left(x^{n-1}\right) \geq P_{0}\left(x^{n-1}\right), \forall x^{n-1}, \\
& \beta P_{0}\left(x^{n-1}\right) \geq P_{1}\left(x^{n-1}\right), \forall x^{n-1},
\end{aligned}
$$


imply

$$
\begin{aligned}
& \beta P_{1}\left(x^{n}\right) \geq P_{0}\left(x^{n}\right), \quad \forall x^{n}, \\
& \beta P_{0}\left(x^{n}\right) \geq P_{1}\left(x^{n}\right), \quad \forall x^{n} .
\end{aligned}
$$

\section{DOES THERE EXISTS A POST CHANNEL FOR WHICH FEEDBACK INCREASES THE CAPACITY?}

For the nonfeedback capacity we do not have an analytical expression, but only the infinite letter expression given in 19. In general, for any finite state channel we have the following upper bounds [20, Theorem 4.6.1] [19, Theorem 15]

$$
C \leq \frac{1}{n} \max _{s_{0}} \max _{P\left(x^{n}\right)} I\left(X^{n} ; Y^{n} \mid s_{0}\right)+\frac{\log |\mathcal{S}|}{n},
$$

for any integer $n \geq 1$. The notation $|\mathcal{S}|$ refers to the number of channel states. However, for the POST channel we have a tighter upper bound given in the following corollary.

Lemma 13 (Upper bound on the capacity of the POST channel): For any POST channel the following upper bound holds

$$
C \leq \frac{1}{n} \max _{s_{0}} \max _{P\left(x^{n}\right)} I\left(X^{n} ; Y^{n} \mid s_{0}\right)
$$

for any integer $n \geq 1$.

Proof: The proof of this upper bound follows from the subadditivity of the sequence

$$
\left\{\max _{s_{0}} \max _{P\left(x^{N}\right)} I\left(X^{N} ; Y^{N} \mid s_{0}\right)\right\}_{N \geq 1} .
$$

In [20, Theorem 4.6.1] or in [19, Theorem 16] it is proved that the sequence $\left\{\max _{s_{0}} \max _{P\left(x^{N}\right)} I\left(X^{N} ; Y^{N} \mid s_{0}\right)\right\}_{N \geq 1}+\log |\mathcal{S}|$ is subadditive. We note that for the POST channel the conditioning on $S_{n}$ done in [20, (4A.26)] or in [19, (67)] is not needed since $S_{n}$ is a function of $Y_{n}$. Because of the subadditivity it follows that the following limit exists and satisfies

$$
\lim _{N \rightarrow \infty} \max _{s_{0}} \max _{P\left(x^{N}\right)} I\left(X^{N} ; Y^{N} \mid s_{0}\right)=\inf _{N} \max _{s_{0}} \max _{P\left(x^{N}\right)} I\left(X^{N} ; Y^{N} \mid s_{0}\right) .
$$

From Fano's inequality it follows that the capacity is upper bounded by this limit and from 99) it follows that $\max _{s_{0}} \max _{P\left(x^{N}\right)} I\left(X^{N} ; Y^{N} \mid s_{0}\right)$ upper bound the capacity for any $N \geq 1$.

Given the results that feedback does not increase the capacity of a $\operatorname{POST}(a, b)$, the question naturally arises: does there exist a specific POST channel where feedback strictly increases the capacity? The answer is affirmative. The idea is to find a POST channel that consists of two states such that when there is feedback, the optimal input distributions given the states differ significantly among the different states. For the binary DMC it was shown in [23] and independently in [24] that the input probability that achieves the capacities is in $\left[\frac{1}{e}, 1-\frac{1}{e}\right]$ for each of the two alphabet symbols. Hence, in the case of a binary post channel with feedback the optimal input probability as a function of the state will not vary too much. But as we increase the alphabet size we can construct a POST channel where the optimal input probabilities as a function of the state would vary significantly. Such a channel is presented in Fig. 6 

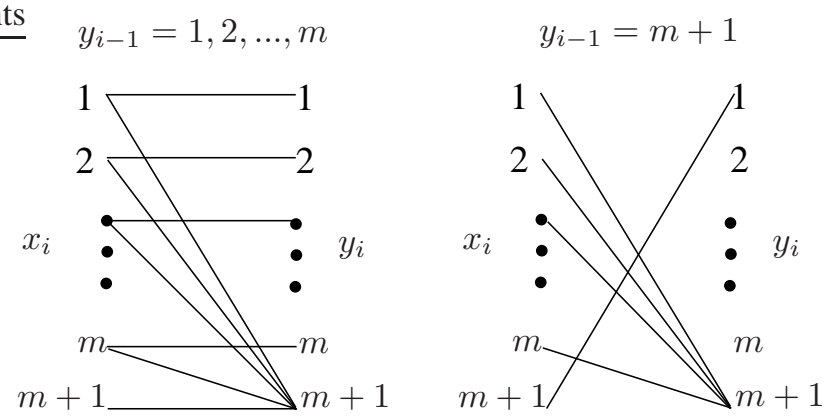

Fig. 6. A POST channel where feedback increases capacity when $m$ is large. The probability associated with each edge is either $\frac{1}{2}$ or 1 .

TABLE III

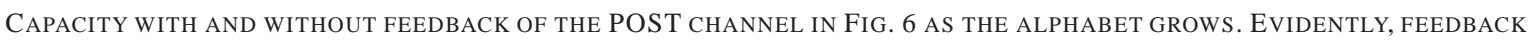
INCREASES CAPACITY FOR SOME VALUES OF $m$

\begin{tabular}{||c|c|c|c||}
\hline \hline$m$ & $\begin{array}{c}\text { upper bound on capacity } \\
\frac{1}{6} \max _{s_{0}} \max _{P\left(x^{6}\right)} I\left(X^{6} ; Y^{6} \mid s_{0}\right)\end{array}$ & $\begin{array}{c}\text { lower bound on feedback capacity } \\
R=\frac{\log _{2} m}{3}\end{array}$ & $\begin{array}{c}\text { feedback capacity } \\
100\end{array}$ \\
\hline $2^{0}$ & 0.7918 & 0 & 0.7595 \\
\hline $2^{1}$ & 0.8568 & 0.3333 & 0.8325 \\
\hline $2^{2}$ & 0.9803 & 0.6667 & 1.0000 \\
\hline $2^{3}$ & 1.1711 & 1.0000 & 1.2599 \\
\hline $2^{4}$ & 1.3865 & 1.3333 & 1.5366 \\
\hline $2^{5}$ & 1.6098 & 1.6667 & 1.8260 \\
\hline $2^{6}$ & 1.8374 & 2.0000 & 2.1252 \\
\hline $2^{7}$ & 2.0683 & 2.3333 & 2.4319 \\
\hline $2^{8}$ & 2.3019 & 2.6667 & 2.7444 \\
\hline $2^{9}$ & 2.5376 & 3.0000 & 3.06140 \\
\hline $2^{10}$ & 2.7751 & 3.3333 & 3.3818 \\
\hline \hline
\end{tabular}

We can determine the feedback capacity analytically using the following lemma, which is proved in Appendix E.

Lemma 14: The feedback capacity of the channel in Fig. 6 is given by,

$$
C_{f b}=\max _{\gamma, \delta \in[0,1]}\left\{\frac{2 \delta}{2 \delta+1+\gamma}\left(\frac{\bar{\gamma}}{2} \log _{2}(m)+h_{2}\left(\frac{1+\gamma}{2}\right)-(1-\gamma)\right)+\frac{1+\gamma}{2 \delta+1+\gamma}\left(h_{2}(\delta)\right)\right\}
$$

Corollary 15: Note that as $m$ approaches infinity, $\frac{\bar{\gamma}}{2} \log _{2}(m)+h_{2}\left(\frac{1+\gamma}{2}\right)-(1-\gamma) \simeq \frac{\bar{\gamma}}{2} \log _{2}(m)>>h_{2}(\delta)$, thus $C_{f b} \simeq \max _{\gamma \in[0,1]}\left\{\frac{1-\gamma}{3+\gamma} \log _{2}(m)\right\}=\frac{\log _{2}(m)}{3}$.

This gives us the intuition to suggest the following simple scheme approximately capacity-achieving for large $m$. If $y_{i-1} \leq m$, then transmit $\log _{2}(m)$ bits via input $X_{i}=1,2, \ldots, m$. The probability that these bits would be received at the decoder is $\frac{1}{2}$. If $y_{i-1}=m+1$, then $X_{i}=m+1$. Thus, the rate transmitted error free for this 
scheme is

$$
R=\frac{\text { average bit transmitted }}{\text { average usage of channels }}=\frac{\frac{1}{2} \log _{2} m+\frac{1}{2} 0}{\frac{1}{2} 1+\frac{1}{2} 2}=\frac{\log _{2} m}{3} .
$$

We clearly see from Table $\amalg$ that for $m \geq 2^{2}$ the feedback capacity is strictly larger than the non feedback capacity. The difference increases with $m$.

\section{CONCLUSION AND FURTHER RESEARCH}

We have introduced and studied the family of POST channels and showed, somewhat surprisingly, that feedback does not increase the capacity of the general $\operatorname{POST}(a, b)$ channel. The proof is based on finding the output probability that is induced by the input causal conditioning pmf that optimizes the directed information when feedback is allowed, and then proving that this output pmf can be also be induced by an input distribution without feedback. There may be a more direct way, that has thus far eluded us, for proving that feedback does not increase the capacity of the Simple POST channel. We hope that the POST channel introduced in this paper will enhance our understanding of capacity of finite state channels with and without feedback, and help us to find simple capacityachieving codes.

\section{ACKNOWLEDGEMENT}

The authors are grateful to Jiantao Jiao who suggested the proof of 82 .

\section{REFERENCES}

[1] R. E. Blahut. Computation of channel capacity and rate-distortion functions. IEEE Trans. Inf. Theory, 18:460-473, 1972.

[2] S. Arimoto. An algorithm for computing the capacity of arbitrary discrete memoryless channels. IEEE Trans. Inf. Theory, 18:14-20, 1972.

[3] C. E. Shannon. Communication in the Presence of Noise. Proceedings of the IRE, 37(1):10-21, January 1949.

[4] M. S. Pinsker. Information and Information Stability of Random Variables and Processes. Izv. Akad. Nauk, Moskva, 1960. in Russian, translated by A. Feinstein in 1964.

[5] F. Alajaji and T. Fuja. Effect of feedback on the capacity of discrete additive channels with memory. In Proceedings ISIT94, Norway, 1994. IEEE.

[6] Y.-H. Kim. Feedback capacity of stationary Gaussian channels. IEEE Trans. Inf. Theory., 56(1):57-85, 2010.

[7] H. H. Permuter, P. Cuff, B. Van Roy, and T. Weissman. Capacity of the trapdoor channel with feedback. IEEE Trans. Inf. Theory, 54(7):3150-3165, 2009.

[8] O. Elishco and H. H. Permuter. Capacity and coding for the Ising channel with feedback. submitted to IEEE Trans. Inf. Theory. Available at arxiv.org/abs/1205.4674, 2012.

[9] A.J. Goldsmith and P.P. Varaiya. Capacity of fading channels with channel side information. IEEE Trans. Inf. Theory, 43(6):1986 -1992, 1997.

[10] J. Chen and T. Berger. The capacity of finite-state Markov channels with feedback. IEEE Trans. Inf. Theory, 51:780-789, 2005.

[11] T. Berger and F. Bonomi. Capacity and zero-error capacity of Ising channels. IEEE Trans. Inf. Theory, 36:173-180, 1990.

[12] H. Asnani, H. H. Permuter, and T. Weissman. To feed or not to feed back. 2010. submitted to IEEE Trans. Inf. Theory. Available at arxiv.org/abs/1011.1607.

[13] S. Boyd and L. Vandenberghe. Convex Optimization. Cambridge University Press, New-York, 2004.

[14] G. Kramer. Directed information for channels with feedback. Ph.D. dissertation, Swiss Federal Institute of Technology (ETH) Zurich, 1998.

[15] J. Massey. Causality, feedback and directed information. Proc. Int. Symp. Inf. Theory Applic. (ISITA-90), pages 303-305, Nov. 1990. 
[16] H. Marko. The bidirectional communication theory- a generalization of information theory. IEEE Trans. on communication, COM21:1335-1351, 1973.

[17] Y.-H. Kim. A coding theorem for a class of stationary channels with feedback. IEEE Trans. Inf. Theory., 25:1488-1499, April, 2008.

[18] S. Tatikonda and S. Mitter. The capacity of channels with feedback. IEEE Trans. Inf. Theory, 55:323-349, 2009.

[19] H. H. Permuter, T. Weissman, and A. J. Goldsmith. Finite state channels with time-invariant deterministic feedback. IEEE Trans. Inf. Theory, 55(2):644-662, 2009.

[20] R. G. Gallager. Information theory and reliable communication. Wiley, New York, 1968.

[21] I. Naiss and H. H. Permuter. Extension of the Blahut-Arimoto algorithm for maximizing directed information. IEEE Trans. Inf. Theory, 59:204-222, 2013.

[22] R. Ash. Information Theory. Wiley, New York, 1965.

[23] N. Shulman and M. Feder. The uniform distribution as a universal prior. IEEE Trans. Inf. Theory., 50(6):1356-1362, 2004.

[24] G. Kumar and A. Manolakos. No input symbol should occur more frequently than 1-1/e. Available at arxiv.org/abs/1203.4865, 2012.

\section{APPENDiX A}

\section{CONCAVITY OF DIRECTED INFORMATION IN $P\left(x^{n} \| y^{n-1}\right)$}

Lemma 16 (Concavity of directed information in $P\left(x^{n} \| y^{n-1}\right)$ ): Directed information $I\left(X^{n} \rightarrow Y^{n}\right)$ is concave in $P\left(x^{n} \| y^{n-1}\right)$ for a fixed $P\left(y^{n} \| x^{n}\right)$.

Proof: We need to show that for $0 \leq \theta \leq 1$

$$
\begin{aligned}
& \sum_{x^{n}, y^{n}}\left(\theta p_{1}\left(x^{n} \| y^{n-1}\right)+\bar{\theta} p_{2}\left(x^{n} \| y^{n-1}\right) p\left(y^{n} \| x^{n}\right) \log \frac{p\left(y^{n} \| x^{n}\right)}{\sum_{x^{n}}\left(\theta p_{1}\left(x^{n} \| y^{n-1}\right)+\bar{\theta} p_{2}\left(x^{n} \| y^{n-1}\right)\right) p\left(y^{n} \| x^{n}\right)}\right. \\
& \geq \sum_{x^{n}, y^{n}} \theta p_{1}\left(x^{n} \| y^{n-1}\right) p\left(y^{n} \| x^{n}\right) \log \frac{p\left(y^{n} \| x^{n}\right)}{\sum_{x^{n}} p_{1}\left(x^{n} \| y^{n-1}\right) p\left(y^{n} \| x^{n}\right)}+\bar{\theta} p_{2}\left(x^{n} \| y^{n-1}\right) p\left(y^{n} \| x^{n}\right) \log \frac{p\left(y^{n} \| x^{n}\right)}{\sum_{x^{n}} p_{2}\left(x^{n} \| y^{n-1}\right) p\left(y^{n} \| x^{n}\right)}
\end{aligned}
$$

This inequality may be written as

$$
\begin{aligned}
& \sum_{x^{n}, y^{n}} \theta p_{1}\left(x^{n} \| y^{n-1}\right) p\left(y^{n} \| x^{n}\right) \log \frac{\sum_{x^{n}} p_{1}\left(x^{n} \| y^{n-1}\right) p\left(y^{n} \| x^{n}\right)}{\sum_{x^{n}}\left(\theta p_{1}\left(x^{n} \| y^{n-1}\right)+\bar{\theta} p_{2}\left(x^{n} \| y^{n-1}\right)\right) p\left(y^{n} \| x^{n}\right)} \\
&+\bar{\theta} p_{2}\left(x^{n} \| y^{n-1}\right) p\left(y^{n} \| x^{n}\right) \log \frac{\sum_{x^{n}} p_{2}\left(x^{n} \| y^{n-1}\right) p\left(y^{n} \| x^{n}\right)}{\sum_{x^{n}}\left(\theta p_{1}\left(x^{n}|| y^{n-1}\right)+\bar{\theta} p_{2}\left(x^{n} \| y^{n-1}\right)\right) p\left(y^{n} \| x^{n}\right)} \geq 0
\end{aligned}
$$

Furthermore,

$$
\begin{aligned}
& \sum_{y^{n}} \theta\left\{\sum_{x^{n}} p_{1}\left(x^{n} \| y^{n-1}\right) p\left(y^{n} \| x^{n}\right)\right\} \log \frac{\sum_{x^{n}} p_{1}\left(x^{n} \| y^{n-1}\right) p\left(y^{n} \| x^{n}\right)}{\sum_{x^{n}}\left(\theta p_{1}\left(x^{n} \| y^{n-1}\right)+\bar{\theta} p_{2}\left(x^{n} \| y^{n-1}\right)\right) p\left(y^{n} \| x^{n}\right)} \\
& +\sum_{y^{n}} \bar{\theta}\left\{\sum_{x^{n}} p_{2}\left(x^{n} \| y^{n-1}\right) p\left(y^{n} \| x^{n}\right)\right\} \log \frac{\sum_{x^{n}} p_{2}\left(x^{n} \| y^{n-1}\right) p\left(y^{n} \| x^{n}\right)}{\sum_{x^{n}}\left(\theta p_{1}\left(x^{n} \| y^{n-1}\right)+\bar{\theta} p_{2}\left(x^{n} \| y^{n-1}\right)\right) p\left(y^{n} \| x^{n}\right)} \geq 0
\end{aligned}
$$

Finally, note that the RHS is a sum of two divergences between pmf's of $y^{n}$ and therefore it is positive.

\section{APPENDIX B}

\section{SUPPORTING INEQUALITIES}

Lemma 17: The inequality

$$
\alpha^{\frac{1}{\bar{\alpha}}} \leq 1
$$


holds for $0 \leq \alpha \leq 1$.

Proof: For $0 \leq \alpha \leq 1$

$$
\log \alpha \leq 0,
$$

which implies

$$
\frac{1}{\bar{\alpha}} \log \alpha \leq 0,
$$

and equivalently

$$
2^{\frac{1}{\alpha} \log \alpha} \leq 2^{0} .
$$

Note that the last inequality is actually (105) for nonnegative $\alpha$.

Lemma 18: The inequality

$$
4 \alpha^{\frac{\alpha+1}{\bar{\alpha}}} \leq 1
$$

holds for $0 \leq \alpha \leq 1$.

Proof: By taking ln of both sides, we need to show

$$
\frac{1+\alpha}{\bar{\alpha}} \ln \alpha+\ln 4 \leq 0,
$$

which is equivalent to

$$
(1+\alpha) \ln \alpha+\bar{\alpha} \ln 4 \leq 0 .
$$

In order to prove (111) we claim that the RHS increases in $\alpha$ for $0 \leq \alpha \leq 1$ and, therefore, the maximum value is obtained at $\alpha=1$ and is 0 . In order to show that the RHS of 111) is increasing we need to show that its derivative is nonnegative, i.e.,

$$
\frac{1}{\alpha}+1+\ln \alpha-\ln 4 \geq 0
$$

or equivalently

$$
1+\alpha+\alpha \ln \alpha-\alpha \ln 4 \geq 0
$$

The RHS of (113) is a convex function and the minimum is obtained when the derivative is zero, i.e.,

$$
1+\ln \alpha+1-\ln 4=0
$$

which implies that $\ln \alpha=\ln \frac{4}{e^{2}}$. Hence, the minimum value of the RHS of 1113$)$ is $1+\frac{4}{e^{2}}+\frac{4}{e^{2}} \ln \frac{4}{e^{2}}-\frac{4}{e^{2}} \ln 4=1-\frac{4}{e^{2}}$, which is positive. Therefore (113) holds, which implies that (111) holds, which implies that (110) holds.

Lemma 19: The following inequality holds

$$
\frac{1+\sqrt{1-4 \alpha^{\frac{\alpha+1}{\bar{\alpha}}}}}{2 \alpha^{\frac{\alpha}{\bar{\alpha}}}} \geq 1
$$

for any $0 \leq \alpha \leq 1$.

Proof: Since $\alpha$ is nonnegative we need to show

$$
\sqrt{1-4 \alpha^{\frac{\alpha+1}{\alpha}}} \geq 2 \alpha^{\frac{\alpha}{\alpha}}-1 .
$$


This would be true if

$$
1-4 \alpha^{\frac{\alpha+1}{\bar{\alpha}}} \geq 4 \alpha^{2 \frac{\alpha}{\bar{\alpha}}}-4 \alpha^{\frac{\alpha}{\bar{\alpha}}}+1
$$

which can be simplified to

$$
1 \geq \alpha^{\frac{\alpha}{\bar{\alpha}}}+\alpha^{\frac{1}{\bar{\alpha}}}
$$

which can be written as

$$
1 \geq \alpha^{\frac{\alpha}{\bar{\alpha}}}(1+\alpha)
$$

When $\alpha \rightarrow 0^{+}$we have equality, hence it suffices to show that the equality holds after taking the derivative with respect to $\alpha$. We use the equality $f^{\prime}(\alpha)=f(\alpha)(\ln f(\alpha))^{\prime}$ to find

$$
\left(\alpha^{\frac{\alpha}{\bar{\alpha}}}\right)^{\prime}=\alpha^{\frac{\alpha}{\bar{\alpha}}}\left(\frac{\ln \alpha}{\bar{\alpha}^{2}}+\frac{1}{\bar{\alpha}}\right) .
$$

Hence, applying the derivative on 119 it remains to show that

$$
\alpha^{\frac{\alpha}{\bar{\alpha}}}\left(\frac{\ln \alpha}{\bar{\alpha}^{2}}+\frac{1}{\bar{\alpha}}\right)(1+\alpha)+\alpha^{\frac{\alpha}{\bar{\alpha}}} \leq 0 .
$$

or more simply

$$
\ln \alpha+\bar{\alpha}+\frac{\bar{\alpha}^{2}}{1+\alpha} \leq 0
$$

Note that if $\alpha=1$ there is equality. Hence it suffices to show that the derivative of the LHS is non-negative for $0 \leq \alpha \leq 1$. I.e.,

$$
\frac{1}{\alpha}-1-\frac{2 \bar{\alpha}}{1+\alpha}-\frac{\bar{\alpha}^{2}}{(1+\alpha)^{2}} \geq 0
$$

which is equivalent to

$$
\frac{\bar{\alpha}}{\alpha}-\frac{\bar{\alpha}(3+\alpha)}{(1+\alpha)^{2}} \geq 0
$$

and this is true if

$$
(1+\alpha)^{2}-\alpha(3+\alpha) \geq 0
$$

which is equivalent to

$$
1-\alpha \geq 0
$$

which is true.

\section{APPENDIX C}

PROOF OF LEMMA 12

Proof: Suppose Eq. 95 holds; for Eq. (96) to hold, we need to have $\forall x^{n-1}$,

$$
\begin{gathered}
\beta\left(b \gamma P_{0}\left(x^{n-1}\right)-\bar{b} P_{1}\left(x^{n-1}\right)\right) \geq a P_{0}\left(x^{n-1}\right)-\bar{a} \gamma P_{1}\left(x^{n-1}\right) \\
\beta\left(-\bar{a} \gamma P_{0}\left(x^{n-1}\right)+a P_{1}\left(x^{n-1}\right)\right) \geq-\bar{b} P_{0}\left(x^{n-1}\right)+b \gamma P_{1}\left(x^{n-1}\right) \\
\beta\left(a P_{0}\left(x^{n-1}\right)-\bar{a} \gamma P_{1}\left(x^{n-1}\right)\right) \geq b \gamma P_{0}\left(x^{n-1}\right)-\bar{b} P_{1}\left(x^{n-1}\right)
\end{gathered}
$$




$$
\beta\left(-\bar{b} P_{0}\left(x^{n-1}\right)+b \gamma P_{1}\left(x^{n-1}\right)\right) \geq-\bar{a} \gamma P_{0}\left(x^{n-1}\right)+a P_{1}\left(x^{n-1}\right)
$$

or equivalently, 127) and (128) become

$$
\begin{aligned}
& (b \gamma \beta-a) P_{0}\left(x^{n-1}\right) \geq(\bar{b} \beta-\bar{a} \gamma) P_{1}\left(x^{n-1}\right) \\
& (\bar{b}-\bar{a} \gamma \beta) P_{0}\left(x^{n-1}\right) \geq(b \gamma-a \beta) P_{1}\left(x^{n-1}\right)
\end{aligned}
$$

and 129) and (130) become

$$
\begin{aligned}
& (\bar{b}-\bar{a} \gamma \beta) P_{1}\left(x^{n-1}\right) \geq(b \gamma-a \beta) P_{0}\left(x^{n-1}\right) \\
& (b \gamma \beta-a) P_{1}\left(x^{n-1}\right) \geq(\bar{b} \beta-\bar{a} \gamma) P_{0}\left(x^{n-1}\right)
\end{aligned}
$$

Because of the similarity of the equations its enough to consider only 131 and 132. Now we will consider a few cases.

The region of $\beta$ that satisfies (131) and (134): we will divide the treatment of (131) (or equivalently (134)) into two cases.

Case 1: $\beta \bar{b}-\bar{a} \gamma>0$ or equivalently $\beta>\frac{\bar{a}}{b} \gamma$; Eq. (131) becomes

$$
P_{0}\left(x^{n-1}\right) \frac{b \gamma \beta-a}{\bar{b} \beta-\bar{a} \gamma} \geq P_{1}\left(x^{n-1}\right) \text {. }
$$

By the assumption of the induction this would be true for all $x^{n-1}$ if

$$
\frac{b \gamma \beta-a}{\bar{b} \beta-\bar{a} \gamma} \geq \beta
$$

or equvalently

$$
\bar{b} \beta^{2}-\gamma(\bar{a}+b) \beta+a \leq 0
$$

This implies

$$
\frac{\gamma(\bar{a}+b)-\sqrt{\gamma^{2}(\bar{a}+b)^{2}-4 a \bar{b}}}{2 \bar{b}} \leq \beta \leq \frac{\gamma(\bar{a}+b)+\sqrt{\gamma^{2}(\bar{a}+b)^{2}-4 a \bar{b}}}{2 \bar{b}},
$$

which is the interval $\mathcal{L}_{1}$ defined in 79 .

Case 2: $\beta \bar{b}-\bar{a} \gamma<0$ or equivalently $\beta<\frac{\bar{a}}{b} \gamma$; Eq. 131 becomes

$$
P_{0}\left(x^{n-1}\right) \frac{b \gamma \beta-a}{\bar{b} \beta-\bar{a} \gamma} \leq P_{1}\left(x^{n-1}\right)
$$

which is true based on the induction assumption if

$$
\frac{b \gamma \beta-a}{\bar{b} \beta-\bar{a} \gamma} \leq \frac{1}{\beta}
$$

This is equivalent to

$$
b \gamma \beta^{2}-(a+\bar{b}) \beta+\bar{a} \gamma \geq 0,
$$

and this is true if

$$
\beta \geq \frac{(a+\bar{b})+\sqrt{(a+b)^{2}-4 \bar{a} b \gamma^{2}}}{2 b \gamma}
$$


which is the interval $\mathcal{L}_{2}$, or

$$
\beta \leq \frac{(a+\bar{b})-\sqrt{(a+\bar{b})^{2}-4 \bar{a} b \gamma^{2}}}{2 b \gamma}
$$

which is the interval $\mathcal{L}_{3}$.

The region of $\beta$ that satisfies (132) and (133): we will divide the treatment of (132) (or equivalently (133) into two cases.

Case 1: $b \gamma-\beta a>0$ or equivalently $\beta<\frac{b \gamma}{a}$; Eq. (132) becomes

$$
P_{0}\left(x^{n-1}\right) \frac{\bar{b}-\bar{a} \gamma \beta}{b \gamma-\beta a} \geq P_{1}\left(x^{n-1}\right) .
$$

By the assumption of the induction this would be true for all $x^{n-1}$ if

$$
\frac{\bar{b}-\bar{a} \gamma \beta}{b \gamma-\beta a} \geq \beta
$$

or equivalently

$$
a \beta^{2}-\gamma(b+\bar{a}) \beta+\bar{b} \geq 0
$$

This implies

$$
\beta \leq \frac{\gamma(\bar{a}+b)-\sqrt{\gamma^{2}(\bar{a}+b)^{2}-4 a \bar{b}}}{2 a},
$$

which is the interval $\mathcal{L}_{4}$, or

$$
\beta \geq \frac{\gamma(\bar{a}+b)+\sqrt{\gamma^{2}(\bar{a}+b)^{2}-4 a b}}{2 a}
$$

which is the interval $\mathcal{L}_{5}$.

Case 2: $b \gamma-\beta a<0$ or equivalently $\beta>\frac{b \gamma}{a}$; Eq. (132) becomes

$$
P_{0}\left(x^{n-1}\right) \frac{\bar{b}-\bar{a} \gamma \beta}{b \gamma-\beta a} \leq P_{1}\left(x^{n-1}\right) .
$$

By the assumption of the induction this would be true for all $x^{n-1}$ if

$$
\begin{gathered}
\frac{\bar{b}-\bar{a} \gamma \beta}{b \gamma-\beta a} \leq \frac{1}{\beta} \\
\beta \bar{b}-\bar{a} \gamma \beta^{2} \geq b \gamma-\beta a \\
\bar{a} \gamma \beta^{2}-\beta(a+\bar{b})+b \gamma \leq 0 .
\end{gathered}
$$

This implies

$$
\frac{(a+\bar{b})-\sqrt{(a+b)^{2}-4 \bar{a} b \gamma^{2}}}{2 \bar{a} \gamma} \leq \beta \leq \frac{(a+\bar{b})+\sqrt{(a+b)^{2}-4 \bar{a} b \gamma^{2}}}{2 \bar{a} \gamma}
$$

which is the interval $\mathcal{L}_{6}$.

\section{APPENDIX D}

INEQUALITIES NEEDED FOR THE $\operatorname{POST}(a, b)$ CHANNEL

In this appendix we prove inequalities that are needed for proving that feedback does not increase the capacity of $\operatorname{POST}(a, b)$ channel. All inequalities contains $\gamma$ which is defined in 80 and obviously $0 \leq a \leq 1$ and $0 \leq b \leq 1$. 
A. $a \geq \bar{b}$ and $a \bar{a} \leq b \bar{b}$ implies that $a \geq b$.

Proof: Let $\rho_{a}=\left|\frac{1}{2}-a\right|$ and $\rho_{b}=\left|\frac{1}{2}-b\right|$. Hence,

$$
\begin{aligned}
a \bar{a} & =\left(\frac{1}{2}-\rho_{a}\right)\left(\frac{1}{2}+\rho_{a}\right) \\
b \bar{b} & =\left(\frac{1}{2}-\rho_{b}\right)\left(\frac{1}{2}+\rho_{b}\right)
\end{aligned}
$$

Since $a \bar{a} \leq b \bar{b}$ it follows that $\rho_{a} \geq \rho_{b}$. And since $a \geq \bar{b}$ it follows that $a=\frac{1}{2}+\rho_{a}$ and this implies $a \geq b$.

B. $\gamma^{2}(\bar{a}+b)^{2}-4 a \bar{b} \geq 0$.

Proof: Assume first that $a \geq \bar{b}$

$$
2^{2 \frac{H(\bar{b})-H(a)}{a-b}} \geq \frac{4 a \bar{b}}{(2-a-\bar{b})^{2}}
$$

Taking $\log$ on both sides we obtain

$$
2 H(\bar{b})-2 H(a) \geq(a-\bar{b})\left(\log 4 \bar{b}+\log a-\log (2-a-\bar{b})^{2}\right)
$$

Note that if $a=\bar{b}$ we have equality, hence it suffices to prove that the inequality holds after applying the derivative with respect to $a$.

$$
-2 \ln \frac{\bar{a}}{a} \geq \ln 4 \bar{b}+\ln a-2 \ln (2-a-\bar{b})+(a-\bar{b})\left(\frac{1}{a}+\frac{2}{2-a-\bar{b}}\right)
$$

If $a=\bar{b}$ we note that there is equality hence it suffices to prove that the inequality holds after taking the derivative with respect to $a$.

$$
\frac{2}{a}+\frac{2}{1-a} \geq \frac{1}{a}+\frac{2}{2-a-\bar{b}}+\frac{1}{a}+\frac{2}{2-a-\bar{b}}+(a-\bar{b})\left(-\frac{1}{a^{2}}+\frac{2}{(2-a-\bar{b})^{2}}\right)
$$

After simplifying we obtain

$$
\frac{a-\bar{b}}{a^{2}}+\frac{2}{\bar{a}}+\frac{2 a+6 \bar{b}-8}{(2-a-\bar{b})^{2}} \geq 0
$$

which after simple algebra it yields

$$
\frac{a-\bar{b}}{a^{2}}+2 \frac{(a-\bar{b})(1-\bar{b})}{\bar{a}(2-a-\bar{b})^{2}} \geq 0
$$

and obviously the inequality holds since $a \geq \bar{b}$ and $b \leq 1$.

Now, if we consider $a \leq b$, then the sign $\geq$ in 156 should be replaces by the sign $\leq$. In 157 the sign should be the same, but in (160) the sign should be the opposite again, which is true.

C. $\gamma(\bar{a}+b)-\sqrt{\gamma^{2}(\bar{a}+b)^{2}-4 a \bar{b}} \leq 2 \bar{b}$, for $a \geq \bar{b}$ and $a \bar{a} \leq b \bar{b}$

Proof: We need to show

$$
\gamma(\bar{a}+b)-2 \bar{b} \leq \sqrt{\gamma^{2}(\bar{a}+b)^{2}-4 a \bar{b}},
$$

since the RHS is nonnegative it suffices to show that

$$
\gamma^{2}(\bar{a}+b)^{2}-4 \bar{b}(\bar{a}+b) \gamma+4 \bar{b}^{2} \leq \gamma^{2}(\bar{a}+b)^{2}-4 a \bar{b},
$$


which simplifies to

$$
\gamma(\bar{a}+b) \geq a+\bar{b}
$$

After applying log on both sides, we need to show that

$$
H(b)-H(a) \geq(a-\bar{b})(\log (a+\bar{b})-\log (\bar{a}+b))
$$

If $a=\bar{b}$ we have equality, hence it suffices to show that after we take derivative with respect to $a$ the inequality holds, i.e.,

$$
\ln \frac{a}{\bar{a}} \geq \log \frac{a+\bar{b}}{\bar{a}+b}+\frac{a-\bar{b}}{a+\bar{b}}+\frac{a-\bar{b}}{\bar{a}+b}
$$

Again, if $b=\bar{a}$ we have equality, and we take the derivative with respect to $b$, i.e.,

$$
0 \leq-\frac{1}{a+\bar{b}}-\frac{1}{\bar{a}+b}+\frac{2 a}{(a+\bar{b})^{2}}+\frac{2 \bar{a}}{(\bar{a}+b)^{2}}
$$

which after simplification equals to

$$
0 \leq \frac{a-\bar{b}}{(a+\bar{b})^{2}}+\frac{\bar{a}-b}{(\bar{a}+b)^{2}}
$$

and since $a-\bar{b}=b-\bar{a}$, we only need to show that

$$
\frac{1}{(a+\bar{b})^{2}} \leq \frac{1}{(\bar{a}+b)^{2}}
$$

which is true since $a+1-b \geq 1-a+b$ when $a \geq b$ and this follows as shown in Appendix D-A

D. $\frac{b \gamma}{a} \geq 1$ for $a \geq \bar{b}$

Proof: We need to show that

$$
-b \log b-\bar{b} \log \bar{b}+a \log a+\bar{a} \log \bar{a} \geq(a-\bar{b})(\log a-\log b)
$$

If $a=\bar{b}$, then both sides equal to zero. Hence, its suffices to show that the inequality holds after applying the derivative with respect to $a$.

$$
\ln \frac{a}{\bar{a}} \geq(\ln a-\log b)+1-\frac{\bar{b}}{a}
$$

We need to show that for $b \geq \bar{a}$

$$
\log \frac{b}{\bar{a}}+\frac{\bar{b}}{a}-1 \geq 0
$$

If $b=\bar{a}$, then equality holds, hence its enough to show that the derivative with respect to $b$ is positive. Namely,

$$
\frac{1}{b}-\frac{1}{a} \geq 0
$$

This is true since we also have $a \bar{a} \leq b \bar{b}$ and it implies $a \geq b$ as shown in Appendix D-A. 
E. $\gamma^{2} \leq \frac{a^{2}}{b \bar{a}}$ for $a \geq \bar{b}$

Proof: Taking log on both sides we need to show

$$
\frac{2 h(b)-2 h(a)}{a-\bar{b}} \leq 2 \log a-\log b-\log \bar{a} .
$$

After simple algebra we obtain

$$
(-b-\bar{a}) \log b-2 \bar{b} \log \bar{b}+2 \bar{b} \log a+(\bar{a}+b) \log \bar{a} \leq 0
$$

We need to show that the inequality holds for $a \geq \bar{b}$. Note that when $a=\bar{b}$. we obtain equality. Therefore its enough to show that the derivative of RHS with respect to $a$ is negative and therefore decreasing from 0 . At this point we transform all the log to be natural base.

$$
\ln b+2 \frac{\bar{b}}{a}-\ln \bar{a}-1-\frac{b}{\bar{a}} \leq 0
$$

Again if $b=\bar{a}$ we obtain equality. Now we take derivative with respect to $b$ and need to show that its negative.

$$
\frac{1}{b}-\frac{2}{a}-\frac{1}{\bar{a}} \leq 0
$$

This is true since $b \geq \bar{a}$.

F. $\frac{\gamma(\bar{a}+b)}{2 b} \geq 1$ for $a \geq \bar{b}$ and $a \bar{a} \leq b \bar{b}$

Proof: We would like to show

$$
\frac{\gamma(\bar{a}+b}{2 \bar{b}} \geq 1
$$

Equivalently after taking $\log$ on both sides,

$$
\begin{gathered}
\frac{h(b)-h(a)}{a-\bar{b}} \geq \log \frac{2 \bar{b}}{\bar{a}+b}, \\
-b \log b-\bar{b} \log \bar{b}+a \log a+\bar{a} \log \bar{a} \\
\geq(a-\bar{b})(\log 2+\log \bar{b}-\log (\bar{a}+b)),
\end{gathered}
$$

Note that if $a=\bar{b}$ we have equality, hence it suffices to show that for $b \geq \bar{a}$ the inequality holds after taking the derivative with respect to $b$, i.e.,

$$
\ln \frac{\bar{b}}{b} \geq \ln 2+\ln \bar{b}-\ln (\bar{a}+b)+(a-\bar{b})\left(-\frac{1}{\bar{b}}-\frac{1}{\bar{a}+b}\right)
$$

If $a=\bar{b}$ we get 0 on both sides. Hence, it suffices to show that in we take derivative with respect to $a$ (179) holds.

$$
0 \geq \frac{1}{\bar{a}+b}-\frac{1}{\bar{b}}-\frac{1}{\bar{a}+b}-\frac{a-\bar{b}}{(\bar{a}+b)^{2}}
$$

and this trivially holds since the RHS is negative while the LHS is 0. 
G. $\gamma \geq \frac{\bar{b}}{b}$ and $\gamma \leq \frac{a}{\bar{a}}$

Proof: Recall $\gamma=2^{\frac{H(b)-H(a)}{a+b-1}}$. We prove only $\gamma \geq \frac{\bar{b}}{b}$ and $\gamma \leq \frac{a}{\bar{a}}$ follows from identical steps just replacing $a$ with $b$.

We need to show that

$$
b 2^{\frac{H(b)}{a+b-1}} \geq \bar{b} 2^{\frac{H(a)}{a+b-1}} .
$$

Equivalently,

$$
\begin{gathered}
\log b+\frac{H(b)}{a+b-1} \geq \log \bar{b}+\frac{H(a)}{a+b-1} . \\
(a+b-1) \log b-b \log b-\bar{b} \log \bar{b} \geq(a+b-1) \log \bar{b}-a \log a-\bar{a} \log \bar{a} . \\
-\bar{a} \log b \geq a \log \bar{b}-a \log a-\bar{a} \log \bar{a} . \\
\bar{a} \log \frac{\bar{a}}{b}+a \log \frac{a}{\bar{b}} \geq 0 .
\end{gathered}
$$

Note that the last equality is a divergence expression between two Bernoulli distributions with parameters $a$ and $\bar{b}$ and hence it's non negative.

\section{APPENDIX E}

Proof of Lemma 14

Proof: By symmetry, for any optimal distribution we will have, $P\left(X_{i}=m+1 \mid Y_{i-1}=k\right)$ equal for all $k \in\{1, \cdots, m\}$. Hence define

$$
\begin{aligned}
& \gamma \triangleq P\left(X_{i}=m+1 \mid Y_{i-1}=k\right) \forall k \in\{1, \cdots, m\} \\
& \delta \triangleq P\left(X_{i}=m+1 \mid Y_{i-1}=m+1\right) .
\end{aligned}
$$

Also, by symmetry, $P\left(X_{i}=l \mid Y_{i-1}=k\right)=\frac{\bar{\gamma}}{m} \forall k, l \in\{1, \cdots, m\}$ and $P\left(X_{i}=l \mid Y_{i-1}=m+1\right)=\frac{\bar{\delta}}{m} \forall l \in$ $\{1, \cdots, m\}$. Thus we have the following transition kernel for the induced output Markov Chain,

$$
\begin{aligned}
P\left(Y_{i}=m+1 \mid Y_{i-1}=m+1\right) & =1-\delta \\
P\left(Y_{i}=m+1 \mid Y_{i-1}=k\right) & =\frac{1+\gamma}{2} \forall k \in\{1,2, \cdots, m\} \\
P\left(Y_{i}=1 \mid Y_{i-1}=m+1\right) & =\delta \\
P\left(Y_{i}=k \mid Y_{i-1}=m+1\right) & =0 \forall k \in\{2, \cdots, m\} \\
P\left(Y_{i}=k \mid Y_{i-1}=l\right) & =\frac{\bar{\gamma}}{2 m} \forall k, l \in\{1,2, \cdots, m\} .
\end{aligned}
$$

Define the stationary distribution by $\pi_{k} \triangleq P\left(Y_{i}=k\right)$, for all $k \in\{1, \cdots, m+1\}$. To obtain the stationary distribution, we have the following balance equations,

$$
\sum_{k=1}^{m+1} \pi_{k}=1
$$




$$
\begin{aligned}
\pi_{m+1} \delta & =\left(\sum_{k=1}^{m} \pi_{k}\right) \frac{1+\gamma}{2} \\
\pi_{1}\left(1-\frac{\bar{\gamma}}{2 m}\right) & =\pi_{m+1} \delta+\left(\sum_{k=2}^{m} \pi_{k}\right) \frac{\bar{\gamma}}{2 m} \\
\pi_{k}\left(1-\frac{\bar{\gamma}}{2 m}\right) & =\left(\sum_{l=1, l \neq k}^{m} \pi_{l}\right) \frac{\bar{\gamma}}{2 m} \forall k \in\{2, \cdots, m\} .
\end{aligned}
$$

We solve for the Case: $\mathbf{m}=\mathbf{1}$, first, for which the equations are,

$$
\begin{aligned}
\pi_{1}+\pi_{2} & =1 \\
\pi_{2} \delta & =\pi_{1} \frac{1+\gamma}{2},
\end{aligned}
$$

which yields, $\pi_{1}=\frac{\delta}{\delta+\frac{1+\gamma}{2}}$ and $\pi_{2}=\frac{\frac{1+\gamma}{2}}{\delta+\frac{1+\gamma}{2}}$. Now note,

$$
\begin{aligned}
I\left(X_{i} ; Y_{i} \mid Y_{i-1}\right) & =\pi_{1}\left(H\left(Y_{i} \mid Y_{i-1}=1\right)-H\left(Y_{i} \mid X_{i}, Y_{i-1}=1\right)\right)+\pi_{2}\left(H\left(Y_{i} \mid Y_{i-1}=2\right)-H\left(Y_{i} \mid X_{i}, Y_{i-1}=2\right)\right) \\
& =\pi_{1}\left(h_{2}\left(\frac{1+\gamma}{2}\right)-(1-\gamma)\right)+\pi_{2} h_{2}(\delta) .
\end{aligned}
$$

Now, as $C_{f b}=\max _{\gamma, \delta \in[0,1]} I\left(X_{i} ; Y_{i} \mid Y_{i-1}\right)$, we obtain

$$
\begin{aligned}
C_{f b} & =\max _{\delta, \gamma \in[0,1]}\left\{\frac{\delta}{\delta+\frac{1+\gamma}{2}}\left(h_{2}\left(\frac{1+\gamma}{2}\right)-(1-\gamma)\right)+\frac{\frac{1+\gamma}{2}}{\delta+\frac{1+\gamma}{2}}\left(h_{2}(\delta)\right)\right\} \\
C_{f b} & =\max _{\delta, \gamma \in[0,1]}\left\{\frac{2 \delta}{2 \delta+1+\gamma}\left(h_{2}\left(\frac{1+\gamma}{2}\right)-(1-\gamma)\right)+\frac{1+\gamma}{2 \delta+1+\gamma}\left(h_{2}(\delta)\right)\right\} .
\end{aligned}
$$

Now, we deal with general Case: $m \geq 2$, where from the symmetry in balance equations, $\pi_{k}$ are equal $\forall k \in$ $\{2, \cdots, m\}$, and hence we obtain the following on solving the balance equations,

$$
\begin{aligned}
\pi_{1} & =A \frac{(m-1) \gamma+m+1}{1-\gamma} \\
\pi_{k} & =A \forall k \in\{2, \cdots, m\} \\
\pi_{m+1} & =A \frac{(1+\gamma)(m)}{\delta(1-\gamma)},
\end{aligned}
$$

where constant $A=\frac{\delta(1-\gamma)}{2 m \delta+m(1+\gamma)}$. Note that $I\left(X_{i} ; Y_{i} \mid Y_{i-1}=k\right)=h_{2} \underbrace{\left(\frac{\bar{\gamma}}{2 m}, \cdots, \frac{\bar{\gamma}}{2 m}\right.}_{\text {times }}, \frac{1+\gamma}{2})-(1-\gamma) \forall k \in$ $\{1, \cdots, m\}$. Also note that $I\left(X_{i} ; Y_{i} \mid Y_{i-1}=m+1\right)=h_{2}(\delta)$. Thus we obtain

$$
\begin{aligned}
I\left(X_{i} ; Y_{i} \mid Y_{i-1}\right) & =\left(\sum_{k=1}^{m} \pi_{m}\right)\left(h_{2}(\underbrace{\frac{\bar{\gamma}}{2 m}, \cdots, \frac{\bar{\gamma}}{2 m}}_{m \text { times }}, \frac{1+\gamma}{2})-(1-\gamma)\right)+\pi_{m+1} h_{2}(\delta) \\
& \left.=\frac{2 \delta}{2 \delta+1+\gamma}(h_{2} \underbrace{\frac{\bar{\gamma}}{2 m}, \cdots, \frac{\bar{\gamma}}{2 m}}_{m \text { times }}, \frac{1+\gamma}{2})-(1-\gamma)\right)+\frac{1+\gamma}{2 \delta+1+\gamma}\left(h_{2}(\delta)\right) .
\end{aligned}
$$


Now, as $C_{f b}=\max _{\gamma, \delta \in[0,1]} I\left(X_{i} ; Y_{i} \mid Y_{i-1}\right)$, after some basic algebraic manipulation we obtain the following expression:

$$
C_{f b}=\max _{\delta, \gamma \in[0,1]}\left\{\frac{2 \delta}{2 \delta+1+\gamma}\left(\frac{\bar{\gamma}}{2} \log _{2}(m)+h_{2}\left(\frac{1+\gamma}{2}\right)-(1-\gamma)\right)+\frac{1+\gamma}{2 \delta+1+\gamma}\left(h_{2}(\delta)\right)\right\} .
$$

